\title{
MULTI-CRITERIA OPTIMIZATION OF TRAFFIC SIGNALS: MOBILITY, SAFETY, AND ENVIRONMENT
}

\author{
Aleksandar Stevanovic ${ }^{a^{*}}$, Jelka Stevanovic $^{\mathrm{b}}$, Jaehyun So $^{\mathrm{c}}$, Marija Ostojic ${ }^{\mathrm{d}}$ \\ ${ }^{a}$ Assistant Professor, Department of Civil, Environmental and Geomatics Engineering, Florida Atlantic \\ University, 777 Glades Rd, Bldg 36, Rm 225, Boca Raton, FL 33431, USA, astevano@ fau.edu. \\ ${ }^{\mathrm{b}}$ Independent Consultant, 2145 NW $3{ }^{\text {rd }}$ CT, Boca Raton, FL 33431, USA, jelkastev@yahoo.com. \\ ${ }^{\mathrm{c}}$ Post-doctoral Scholar, Department of Civil, Geo, and Environmental Engineering, Technische \\ Universität München, Arcisstrasse 21, 80333 München, Germany, ssojae82@ gmail.com. \\ ${ }^{\mathrm{d}}$ Graduate Research Assistant, Department of Civil, Environmental and Geomatics Engineering, Florida \\ Atlantic University, 777 Glades Rd, Bldg 4, Rm 101, Boca Raton, FL 33431, USA, \\ mostojic2013@my.fau.edu.
}

\begin{abstract}
Two-dimensional multi-objective optimizations have been used for decades for the problems in traffic engineering although only few times so far in the optimization of signal timings. While the other engineering and science disciplines have utilized visualization of 3-dimensional Pareto fronts in the optimization studies, we have not seen many of those concepts applied to traffic signal optimization problems. To bridge the gap in the existing knowledge this study presents a methodology where 3-dimensional Pareto Fronts of signal timings, which are expressed through mobility, (surrogate) safety, and environmental factors, are optimized by use of an evolutionary algorithm. The study uses a segment of 5 signalized intersections in West Valley City, Utah, to test signal timings which provide a balance between mobility, safety and environment. In addition, a set of previous developed signal timing scenarios, including some of the Connected Vehicle technologies such as GLOSA, were conducted to evaluate the quality of the 3dimensional Pareto front solutions. The results show success of 3-dimensinal Pareto fronts moving towards optimality. The resulting signal timing plans do not show large differences between themselves but all improve on the signal timings from the field, significantly. The commonly used optimization of standard single-objective functions shows robust solutions. The new set of Connected Vehicle technologies also shows promising benefits, especially in the area of reducing inter-vehicular friction. The resulting timing plans from two optimization sets (constrained and unconstrained) show that environmental and safe signal timings coincide but somewhat contradict mobility. Further research is needed to apply similar concepts on a variety of networks and traffic conditions before generalizing findings.
\end{abstract}

Keywords: traffic signals, multi-objective optimization, safety, environment, simulation, 3-D visualization, fuel consumption, emission modeling, surrogates, evolutionary algorithms. 


\section{INTRODUCTION}

Externality is defined, in economics, as the cost or benefit that affects a party who did not choose to incur that cost or benefit. We can also think of externalities, as positive or negative byproducts of certain processes. In transportation, and more specifically roadway traffic, examples of negative externalities are safety incidents/crashes, air pollution, noise, etc. Although byproducts of human activity to move people and freight around, which is a broad definition of transportation, these externalities are not less important than the mobility metrics (e.g. travel time, delay, speed, number of completed trips) that we usually use to qualify success of a transportation system/process.

In traffic engineering design, and more specifically in the operations of signalized intersections, some of these externalities (e.g. fuel consumption which is directly related to environment/air pollution), have been used to decide how to meaningfully tradeoff between mobility performance metrics such as delay and number of stops (Robertson et al., 1980). Since development of externality metrics (e.g. fuel consumed or potential conflicts created) requires methodology that usually goes beyond capabilities of most of the driver-behavior-based traffic simulation models, integration of simulation models and outside externality models have been a natural way to include externalities in the traffic engineering optimization processes (Stevanovic et al., 2009).

Logically, since complex stochastic simulation models require evolutionary algorithms to derive (near) optimal solutions (Foy et al., 1992; Park et al., 1999), the field of transportation engineering has seen a significant number of studies where Genetic Algorithms (GAs), and other evolutionary processes, are embedded in the optimization framework to reduce negative mobility and externality performance metrics (Oda et al. 2004 and Tan et al., 2012). The next step were multi-objective optimization studies, where firstly competing multiple objectives were integrated in the single objective function, followed by an outburst of studies where Pareto Fronts (2dimensional objective functions) were developed to address optimization of mobility and externality performance metrics ( $\mathrm{Li}$ et al., 2004; Kwak et al., 2012; Ma, 2012). While the other engineering science disciplines went a step further to utilize (and visualize), 3-dimensional Pareto fronts, we have not seen many of those concepts in transportation and specifically not applied to traffic signal optimization problems.

To summarize the gap in the existing studies - there has not been a study that presents 3dimensional optimization of traffic signals (signal timings) based on their influence on mobility, safety, and environmental factors. Objective of this article is to present such an approach where 3-dimensional Pareto Fronts of signal timing solutions, which are expressed through throughputs (measure of mobility), conflicts (surrogate measure of safety), and fuel consumption (measure of environmental impacts), are optimized by use of an evolutionary algorithm in the stochastic optimization environment.

This study builds upon similar 2-dimensional optimization studies (Chen and Yu, 2007; Ma 2012; Stevanovic et al., 2013a etc.) and proposes a novel approach to integrating the evaluation of mobility metrics, surrogate measures of safety, fuel consumption, and stochastic optimization of signal timings. VISSIM microsimulation software (PTV, 2010), Surrogate Safety Assessment Model (SSAM) (Gettman et al., 2008), Comprehensive Modal Emission Model (CMEM) (Barth et al., 2000), and VISSIM-based Genetic Algorithm for Optimization of Signal 
Timings (VISGAOST) (Stevanovic et al., 2007) have been integrated in a framework to optimize performance of traffic signals by taking in consideration 3-dimensioanl nature of traffic operations (mobility, safety, and environment). The optimization process moves through the 3dimensional space and generates surfaces which improve always at least one of the objective criteria, without worsening the other two.

The final result is a 3-dimenisonal Pareto front surface that gives system users a chance to select between points (signal timing plans) on the surface, understanding that a sacrifice that has to be made (by preferring improvement in a particular objective criterion over the other two) is the smallest that one can get (assuming that the optimization process led to a (near) optimal solution). The optimization experiments are divided into two parts, where the first part explores a variety of solutions in a non-constrained approach, thus representing a more theoretical approach to the problem. The second part constraints mobility metrics in such a way that derived solutions do not impede mobility of the system by much, thus representing more of a practical approach to the retiming of traffic signals. The authors investigate also how few common single-objectiveoptimization scenarios compare to the derived 3-dimensional Pareto front surfaces.

\section{LITERATURE REVIEW}

Research described in this paper includes a variety of concepts and methods that have been previously addressed in the work of others, including: integration of safety and environmental measures within signal timing processes, evolutionary algorithms, stochastic optimizations, multi-criteria optimizations and others. To fairly summarize findings from variety of previous studies on these subjects, the authors divide the literature review section into several subsections. It should be noted that the list of the studies cited is not inclusive and it presents a summary of some of the most notable studies in each category. The authors pay special attention to 3dimensional (3-D) multi-criteria optimizations, with potential 3-D visualizations, because such studies were not previously conducted in the area of traffic signal optimization.

One should note that this research study does not consider the re-routing of vehicles due to optimization of signal timing settings; routes are assumed from 'a priori' static route assignment. However, integration of dynamic re-routing and signal optimization problem is a very relevant approach to investigate realistic externalities of traffic signal operations. A reader should, for example, see work of Gartner and Al-Malik (1996) who investigated the problem of traffic flow re-routing induced by traffic signal optimization. Also,Chen and Ben-Akiva, in 1998, considered the interaction of dynamic traffic control and dynamic traffic assignment and proposed a theoretical game method as the solution for a combined dynamic traffic controlassignment problem. More recently, Paz and Chiu (2011) proposed a practical and efficient solution in the form of an adaptive traffic control model. In a single-loop algorithmic structure dynamic assignment flows and demand-responsive signal timing settings were generated simultaneously, at every iteration of the solution algorithm. 


\subsection{Stochastic and Evolutionary Algorithms in Traffic Signal Optimizations}

Use of the Genetic Algorithms (GA), as a form of evolutionary algorithms that use stochastic optimizations, in traffic signal optimization was introduced by Foy et al. (1992). They used a GA to optimize phase sequence and green time splits on a traffic network of four intersections. The network delay, extracted from a simplistic traffic flow simulation model, was minimized with a conclusion that the GA can find near optimal signal timings.

Hadi and Wallace (1993) combined GAs with TRANSYT-7F to optimize all four of the signal timing variables. Their GA optimized phase sequence and cycle length while TRANSYT$7 \mathrm{~F}$ optimized green splits and offsets. They found $15-44 \%$ improvement in system-wide progression on the three case-study networks.

Abu-Lebdeh and Benekohal (1997) considered the GA for signal control in a dynamic environment. It was found that the technique was not ready for online implementation due to the extensive computational time required by the GA. In a similar study Girianna and Benekohal (2002) considered multiple conflicting objectives for optimization, however, they were all integrated into single objective function.

Park et al. (1999) were the first to simultaneously optimize all four signal timing parameters using a GA. A mesoscopic traffic simulator evaluated the quality of the GAoptimized timing plans for oversaturated traffic conditions. The performance of the GA-based signal timings was compared with TRANSYT-7F using CORSIM as an evaluator. Their results showed that a GA found better timing plans for low and high demand periods. The study was extended to consider multiple optimization strategies in Park et al. (2000).

Kovvali and Messer (2002) investigated the sensitivity of GA optimization parameters as a way to improve their computational efficiency and to prove it to be a reliable and practical tool. They investigated the impact of various types of the three GA operators and various values of the basic GA optimization parameters. Their results indicated that for a four-signal arterial, simple GA with the following operators performs the best: real representation, elitism, tournament selection and uniform crossover.

Yun and Park (2005) investigated the performance of three heuristic optimization methods (GA, simulated annealing, and OptQuest Engine) and two traditional tools (SYNCHRO and TRANSYT-7F) in the CORSIM environment. The authors investigated the benefits of optimizing the basic signal timing parameters but also controller, detector, and volume-density related settings. Results showed that the timing plans optimized by GA were better than plans from any other optimization method. It was also found that the other settings can further improve the operations of coordinated actuated control.

In most of these studies, GAs are shown to be better at generating timing plans than any other known optimization tools. However, there are few practical applications of GA optimization as they remained computationally demanding. Until recently when considering optimization of traffic signal timings only single GA-based methodologies were implemented successfully. Last decade exploited the implementation of different types of evolutionary algorithms in signal timings optimization. 


\subsection{Multi-Objective Traffic Signal Optimizations}

Multi-objective optimization (Pareto optimum named after Vilfredo Pareto, an Italian scientist who first introduced the concept of non-inferiority ineconomics ) is an area of multiple criteria decision making, concerned with mathematical optimization problems involving simultaneous optimization of more than one objective function (Pareto, 1896).

Multi-objective optimization problems, generally, do not derive a single optimal solution satisfying each objective. Objective functions are predominantly considered to be conflicting, and as the result there are a number (possibly infinite number of) Pareto optimal solutions quantifying the trade-offs in satisfying these different objectives. That is such a set that any objective value cannot be further improved without worsening another.

Traditional methods for generating the Pareto-optimal set aggregate the objectives into a single objective function: these primarily include weighted method (single scalar vector to optimize), constraint method (preferred objective optimizes, the others treated as constraints), linear programming (considers only linear relationships), goal programming (minimizing deviation from constraints), and others (Cohon, 1978).

Goldberg in 1989 proposed the first GA technique called Pure Pareto ranking approach for solving multi-objective optimization tasks. The approach was based on the Pareto dominance ranking. Fonseca and Fleming (1993) developed a different method called multi-objective GA which penalized the solutions which were dominated by the corresponding densely populated sections of the Pareto front. Horn et al. (1994) build up on Goldberg's research and suggested a Niched Pareto GA for finding the Pareto optimum, which considered the distribution of the population proportionally according to the shared fitness i.e. the fitness of the individual solution in relation to its neighborhood-niche count. The overall goal is to successfully distribute the set of solutions in the search space in relation to the density of the niche. The main drawback in these approaches was the fact that vectors with the same objective function values did not exist which was something that was highly desirable from the users' point of view.

Srinivas and Deb (1994) developed a Non-dominated Sorting Genetic Algorithm (NSGA) which was also based on Goldberg's method with one significant difference: it classified the non-dominated Pareto fronts according to the assigned dummy fitness function and then performed sharing operation. The purpose of this sorting technique was to achieve quick convergence of the population towards non-dominated regions and sharing assured the diversity of the solutions over the entire search area.

Other similar studies by Zitzler and Thiele (1999) (on Strength Pareto Evolutionary Algorithm (SPEA)) and by Knowles and Corne (1999) (on Pareto-Archived Evolution Strategy (PAES)) stressed the importance of maintaining an external population which stored all nondominated solutions discovered in previous steps at every generation. These approaches clearly emphasized the necessity of incorporating elitism in multi-criteria optimization procedures, to prevent the loss of good non-dominated solutions once found. Comparative study by Zitzler et al. (2003) revealed the practical benefit of the elitism feature in the Multi Objective Evolutionary Algorithms.

Deb et al. (2001) developed a non-dominated sorting based multi-objective evolutionary algorithm, which outperformed the other evolutionary algorithms which dedicated more attention to producing diverse Pareto optimal solutions. The following year Deb et al. (2002) presented a 
new version of NSGA-II, which resolved its predecessors' shortcomings in computational complexity, non-elitism approach and the necessity of determining the sharing parameter which guarantied the diversity of the Pareto solution set. In addition, the original concept of Pareto dominance was modified to allow for multiple constrained multiple objective optimization problems to be solved; the benefits of this approach were proven and quantified by comparing it with SPEA and PAES, which represent two of the most recent developments.

Sun et al. (2003) applied NSGA-II to minimize average delay and number of stops where effective green is the design variable with three constraints for an isolated intersection under two-phase control. NSGA-II achieved a close approximation of the optimal Pareto set while using an analytical formula to determine delay and stops.

Abbas et al. (2005) applied NSGA-II to a small three signal network for optimizing the time of day plan scheduling. Abbas and Sharma (2006) build on this research and implemented the same multi-objective optimizer to solve a two-level optimization problem. First, it would select a number of timing plans and then based on grouping traffic states into number of clusters, assign one of the plans to each of the clusters.

Another application of NSGA-II, performed by Branke et al. (2007), was intended for optimization of a traffic-actuated controller. The authors concluded that the algorithm overbearingly outperforms the solutions developed by a traffic engineer with respect to several objectives (travel times, number of stops). NSGA-II was proven to be robust enough to deal with the stochastic nature of the performance parameters returned by the microscopic traffic simulation.

Ma et al. (2010) applied the same algorithm to identify the optimal signal control settings at a two-stage midblock crosswalk, taking into consideration vehicular and pedestrian delay and compared it with the optimization results from Synchro. Similarly, Li et al. (2013) integrated VISSIM and NSGA-II to optimize signal timing plans by maximizing throughput and minimizing average queue. The simulation results indicated that the signal timing plan generated was more efficient in managing the traffic flow at oversaturated intersection than the ones produced by Synchro.

Different types of multi-objective GA were also implemented by other researchers to optimize signal timings, even though the most popular one to date was NSGA. Ceylan and Bell (2004) defined the objective function as the sum of a weighted linear combination of delay and number of stops and by integrating the genetic algorithms, traffic assignment and traffic control solved the equilibrium network design problem simpler than with heuristic algorithms. Girianna and Benekohal (2004) formulated the model proposed as a dynamic optimization problem with the objective of maximizing the total number of vehicles released by the network and penalizing it by queue accumulation along the arterials and used genetic algorithms to find the near optimal signal timing. Kesur (2010) investigated and suggested a multi-objective optimization when there are numerous optimization variables which offered improved optimization efficiency over the standard genetic algorithm. Compared with a single objective optimization for delay minimization strategy, the multi-objective optimizer produced signal timing plans with similar overall delay values, however with considerably more equitable delay distribution.

Chen et al. (2011) presented a multi-objective signal timing optimization model with vehicle volume and the non-motorized transport number as the inputs and traveler delay, stops and traffic capacity as the optimization objectives, and saturation degree is taken as restrictions. 
The multi-objective model was solved by genetic algorithm. Similarly, Lertworawanich (2011) proposed a model which simultaneously optimized intersection spillovers, equity in delays, and system throughputs where the objectives were arranged in an order according to their importance and then this new formulation was solved by genetic algorithms to obtain signal timing plans.

Ceylan (2013) proposed a traffic congestion minimization model in which the traffic signal setting optimization was performed through a combined simulation-optimization model i.e. differential evolutionary optimization technique with the TRANSYT traffic simulation tool and proved to be less computationally demanding than other GAs. Ezzat et al. (2014) aimed at determining a set of optimum/near-optimum signal timings to minimize the total time in the system in oversaturated conditions and their results showed that the evolutionary method for optimization excelled over single objective GA optimizer, when it came to queue lengths and vehicular waiting time (two objective functions).

\subsection{3-D Pareto Front Optimizations in Other Fields}

Newer approaches in generating optimum Pareto solution sets involve various sophisticated algorithms. Different versions of single objective GA and, most recently, various evolutionary algorithms have been developed for determining Pareto optimum. Multi Objective Evolutionary Algorithms (MOEAs) were proven to be applicable in a range of complex scientific and engineering tasks.

Fonseca and Fleming (1993), and Horn et al. (1994) individually reached the same conclusion that as the number of conflicting objective functions increased, more of the search space was expected to be encompassed by Pareto optimality, thus actually making the "theoretical" problem of identifying non-dominated solutions of a multi-objective optimization easier.

Recent advances emphasize the necessity of visually representing Pareto solution sets and focus attention on developing more adequate tools with the purpose of aiding the decision maker's choice. Yukish (2004) developed a hybrid algorithm (Simple Cull and Divide \& Conquer) to identify Pareto frontier in multi-dimensional data sets primarily to facilitate visualization. This algorithm essentially operated two algorithms simultaneously and they were adjusting according to the data set properties.

In addition, Agrawal et al. (2004) proposed a visualization methodology for displaying a Pareto Frontier for more than three objective functions in a multi-objective optimization problem. The intention was to facilitate selection of a potential single optimal design amongst all possible solutions in the Pareto optimum, where every dimension was represented in 2-D or 3-D space. Four years later, similar visualization tool named level diagrams, was developed by Blasco et al. (2008) particularly suited for three or more dimensional Pareto front analysis. Each of these diagrams is representing a single objective and design parameter. This meant that one point of the Pareto front is represented with three points (on 3 diagrams i.e. 3 objectives) at the same position on the $\mathrm{Y}$ axis, while the $\mathrm{X}$ axis corresponds to values of the objective, or decision variables, in physical units.

Madetoja et al. (2008) proposed another visualization method of utilizing computer created environment- virtual reality facilities to display multi-dimensional Pareto fronts, which then can be further analyzed by zooming and rotating the front. It allowed the user to interact 
with the Pareto front and virtually examine the nature of the solutions as well as the interrelationships among the objectives. Similar approach was taken by Bojanowski and Kulak (2011) who developed a Hyper-Radial Visualization (HRV) method to visualize twodimensional data sets in order to assist the decision maker to distinguish between different Pareto solutions.

Ran (2007) following an extensive doctoral degree research, proposed a systematic framework for determination of Weak Pareto Frontier (WPF) solutions instead of Pareto Front (PF) solutions since WPF can provide more compromised design solutions for trade-off than PF (under probabilistic constraints for realistic conceptual design of complex systems). On the other hand, Zou et al. (2007) explored the relationship between a system-level and individual segments' optimal solutions and indicated that local PF points need to lie on the system-level Pareto optimum. The authors proposed a hierarchical optimization method for determining the relationships between system-level and individual objective functions trade-offs, to prevent unrealistic requirements of one level to be applied on the other. The following year, Cevher and Kaplan (2008) implemented a dynamic programming method (as a function of weighted sum) for obtaining an efficient Pareto optimum for determining the number of different types of sensors to deploy for a provided budget. This approach considered multiple objectives such as sensor network utility, lifetime, coverage, etc. while incorporating additional constraints.

Gollub and Vivie-Riedle in 2009 proved the adequacy of implementing a NSGA-II in solving two and three objective functions tradeoff problems with various constraints for vibrational quantum processes. It was pointed out that both 2-D and 3-D Pareto fronts allowed the interaction of various objectives of interest to be clearly quantified. In addition, Sahoo and Albrecht (2010) concluded that Pareto-based ranking for a two-objective optimization problem was more consistent than any other ranking technique. There were also research studies undertaken with the primary goal of finding the most adequate method of approximating the Pareto set of solutions. On the other hand, Holdsworth et al. (2010) proposed a modified MOEA, a hierarchal evolutionary algorithm flexible enough to handle any functional form of individual objectives and provide outputs of different size and diversity.

Diakonikolas (2011) considered the Chord Algorithm for approximation of the Pareto curve as a good approximation of the entire solution set. Same year, Fikse (2011) formulated an approach to accelerate the search of the Pareto optimal set found by a MOGA for Multi-objective traffic network design problems by using approximation techniques. The author used two simple traffic networks to test various combinations of approximation techniques with methods of GA search acceleration (particularly the NSGA-II). It was concluded that the modified algorithm was capable of providing a Pareto front almost equally good as the Pareto front delivered by the original NSGA-II algorithm, but with a much smaller computational effort (reduced by 50\%).

Moreover, Li et al. (2011) implemented an improved NSGA to solve a three-dimensional trade-off problem, which offered solutions with no preference to either of objective functions. Jousselme et al. (2012) utilized a modified NSGA-II for solving a two-objective function which optimized between the network performance quality and the cost of the deployment i.e. number of sensors placed. The algorithm's baseline optimization loop was decomposed to include the human decision and basically to allow the interactivity with an end-user.

Furthermore, Reynoso-Meza et al. (2012) compared three different MOEAs in terms of speed of execution and quality of Pareto optimums produced. Multi Objective Differential 
Evolutionary (MODE) algorithm with spherical pruning (sp-MODE-analyzing the Pareto set identified by using normalized spherical coordinates from a reference point), DE algorithm without archiving strategy and elitist GA were compared and concluded that the sp-MODE outperformed the others.

\subsection{Safety Metrics as Part of Traffic Signal Optimizations}

Until beginning of this century microscopic-simulation-based optimization of signal timings to maximize traffic safety was not a widely researched subject. Few attempts in this area, all of which involved models not commercially available, (Archer and Kosonen 2000, Kosonen and Ree 2000) came to the conclusion that the output data from behavioral models for the road user interactions (i.e. conflicts) should match the corresponding field data.

Drummond et al. (2002) compared actual crash rates for two major arterials in Virginia with operational performance measures derived from Synchro/SimTraffic simulation model. The authors found a high correlation between traffic efficiency performance measures (e.g. delays and stops) from simulation and field crash rates. A year later, Gettman and Head (2003) developed functional requirements and algorithms for a software tool that analyzed surrogate measures of safety generated by a simulation model. This research was the basis for the development of publicly-available SSAM software a few years later (Gettman et al., 2008).

On the other hand, Archer (2004) identified proximal traffic safety indicators time to accident, time to collision, and Post-Encroachment Time which provided a reasonable level of consistency against field collected data, however the simulation model was proved to underestimate the corresponding field values, also particular problem was found to be the general lack of empirical safety indicator data for comparison purpose.

Klunder et al. (2006) proposed the development of a new simulation model aimed to represent driver behavior particularly at intersections with a high level of detail and calibrated with accurate real world data which then generated more accurate safety performance measures. Similarly Ozbay et al. (2008) emphasized the importance of well-calibrated and validated microsimulation models from which surrogate measures of safety could be collected.

Pirdavani et al. (2010) used microsimulation to evaluate safety conditions of signalized 4leg intersections and concluded form the results gathered that driver behavior was highly influenced by increasing speed limits and deterioration in surrogate safety metrics was then observed. Archer's and Young's study (2010) showed that micorsimulation models in VISSIM can be calibrated and validated to match surrogate safety measures (e.g. Time-to-Collision (TTC)) observed at an unsignalized intersection in the field.

Other research studies also corelated the actual crash data with microsimulation-based surrogate safety measures to develop credible crash prediction models, as well as to examine the relationships between real world conflicts and surrogate measures (Son et al., 2011, Park et al., 2011). Tan et al. (2012) developed microscopic simulation model which reasonably represented safety related parameters of signalized intersections which could be used for signal optimization and evaluation of various countermeasures.

Stevanovic et al. (2013a) proposed an integrated approach of VISSIM microsimulation software, SSAM, and VISGAOST to achieve a multi objective optimization of signal timing plans which minimized the number of conflicts while maintaining efficiency of traffic signals. 


\subsection{Environmental Metrics as Part of Traffic Signal Optimizations}

Some of the earliest research on fuel consumption and environmental impacts of signalized intersections dates back to late '70s when Robertson et al. (1980) optimized signal timings using TRANSYT 8 to minimize fuel consumption. They found that when signal timings are not optimized to reduce delays but to reduce total fuel consumption, the benefits of such signal timings may decrease fuel consumption by up to $3 \%$. The fuel consumption was estimated from its linear relationship with traffic performance measures (delay, stops, and average speed) .

This research set an industry standard in optimization of signal timings by defining a Performance Index (PI) as a linear combination of delay and stops which should be minimized to get the minimal fuel consumption. This was one of the first attempts in the field of signal timing optimization to introduce multiple objectives, which in this case were combined in a single optimization function (PI).

Over the next few decades there has been a number of research studies aimed to evaluate environmental impacts of different traffic control strategies (see for example Hallmark and Guensler 1999, Rouphail et al. 2001, Rakha and Ding 2003, Li et al. 2004 etc.). Liao and Machemehl (1996) developed an Analytical Fuel Consumption Model (AFCM), which integrated traffic characteristics, signal control strategies, and roadway geometrical layout to investigate the effects of signal timing on fuel consumption.

Some of the most popular microscopic emission models available today in the US include Motor Vehicle Emission Simulator (MOVES) (Koupal et al. 2002), already mentioned CMEM (Barth et al., 2000) and Virginia Tech Microscopic (VT-Micro) emission model (Rakha et al., 2004), which calculate instantaneous emissions using inputs of vehicle running states. Park et al. (2001) integrated the VISSIM microsimulation model with a speed-based emissions inventory database (MODEM), in order to evaluate the air pollutant levels compared to field conditions measured. In 2003 Nam et al. coupled VISSIM with CMEM to estimate emissions from a single vehicle. CMEM was proved to offer acceptable ranges of error when compared with field measurements.

Progression-based optimization of signal plans in TRANSYT 9, showed significantly reduced amounts of emissions and fuel consumption on an arterial road through an integrated VISSIM-CMEM methodology (Stathopoulos and Noland 2003). The following year, Oda et al. (2004) used a macroscopic model to optimize traffic control settings to reduce CO2. The authors proved high correlation existed between the number of stops and the $\mathrm{CO} 2$ and minimized the number of stops to simplify the calculation burden. Another study by Li et al. (2004) developed a comprehensive performance index function aimed at optimizing cycle length and green time which considered reducing delays, fuel consumption and emissions. The constraint imposed was the minimum pedestrian phase green time.

Another successful integration of microscopic traffic-emission simulation platform (VISSIM and CMEM) was used to evaluate two alternative traffic control strategies and showed that optimal traffic control reduced various pollutant emissions from $3 \%$ to $15 \%$ (Chen and $\mathrm{Yu}$, 2007). Zhou et al. 2008 determined the optimal cycle length and split expected to minimize the vehicle exhaust emissions by utilizing the GA approach and later proved its effectiveness through Matlab GA application on a network consisting of six links.

Stevanovic et al. (2009) integrated VISSIM, CMEM, and VISGAOST for optimizing signal timings in order to achieve minimal fuel consumption and vehicular emissions. In 
addition, Zhang et al. (2009) demonstrated advantages of coordinated signal control over noncoordinated in reducing vehicle pollution by integrating VISSIM-based case study with vehicle specific power metrics VSP-based approach for emissions modelling.

$\mathrm{Ma}$ and Nakamura (2010) proposed a universal emission function for determining the optimal cycle length and green time split that minimized vehicle emissions. The authors concluded that the objective function was mostly appropriate for high heavy vehicle percentage and higher demands in general.

Later in 2012, Ma developed an integrated approach where microscopic traffic simulator was used along with instantaneous emission model and multi-objective evolutionary algorithm for evaluating and improving signal timing plans. The author considered minimizing delay along with energy and environmental consequences by incorporating NSGA-II and clearly demonstrated that different control strategies produced different impacts. Around the same time, Kwak et al. (2012) proposed a traffic signal optimization approach which integrated Transportation Analysis and Simulation System (TRANSIMS) microscopic traffic simulator with VT-Micro model (a microscopic emission and fuel consumption estimation model), and a genetic algorithm -based optimizer. The method identified much better solutions compared to the ones produced by Synchro in terms of air quality, energy and mobility measures.

Furthermore, Robles (2012) used VISSIM outputs to quantify traffic behavior (speed and acceleration) based on various input parameters (demand and geometry). These measures were then inserted into CMEM to calculate emission and fuel consumption. The outputs from VISSIM (delay and average stops) and CMEM are inserted into a multi-objective genetic algorithm (NSGA-II) to determine the solutions which simultaneously minimized total delay, total number of stops and average fuel consumption.

Qian et al. (2013) developed a weighted sum objective function consisting of delay, pollutant emission and traffic capacity which was used to derive the optimal traffic control strategies under different traffic conditions by applying real-coded GA. Zhang et al. (2013) formulated a two-objective optimization function which included delay and exposure to traffic emissions with the goal of optimizing cycle length, offsets, green splits and phase sequences. Simulation based GA was implemented to optimize the objective function.

\subsection{Summary of Related Work}

In summary, researchers have used various evolutionary algorithms (including GAs) in combination with multi-objective optimizations to optimize traffic signals. Various simulation models (ranging from macroscopic to microscopic) have been used in those studies. Also, various surrogate safety and fuel consumption and emission models have been used to convert outputs from driver behavior's simulation models into externalities that can be reduced in the optimization process. Frequently, those studies involved multi-objective optimizations where NGSA or similar evolutionary algorithms were used to identify, save, and progress the Pareto fronts/solutions through the optimization process. Although 3-dimensional multi-objective optimizations have not been so frequent in the transportation engineering filed, multiple studies from the other engineering and science fields have used these approaches. Yet, in spite of all of this great work (in the field of optimization of traffic signals) there has not been a single study where the three most important dimensions of traffic operations at signalized intersections 
(mobility, safety, and environment) have been optimized by adjusting signal timings. This study bridges such a gap in the existing knowledge and offers a solution where, naturally, a 3dimensional Pareto front is utilized by an NSGA-II to identify optimal solutions.

\section{VISSIM-SSAM-VISGAOST FRAMEWORK}

The following subsections describe integration of VISSIM, SSAM, CMEM and VISGAOST to optimize signal timings by taking in consideration mobility, safety and environment.

\subsection{VISSIM}

VISSIM is a microscopic, time-step and behavior-based model developed to simulate urban traffic and public transport operations. VISSIM can analyze traffic operations under variety of operational conditions and it is a well-known tool in traffic engineering community for its ability to replicate real-world conditions. VISSIM uses a psycho-physical driver behavior model developed by Wiedemann (PTV, 2010) whose basic concept is that the driver of a faster moving vehicle decelerates when approaching a slower moving vehicle according to the driver's individual perception threshold. VISSIM is chosen for experiments in this study because it is a tool that was previously integrated with both CMEM and SSAM (Nam et al. 2003; Chen and Yu 2007; Stevanovic et al. 2013a; etc.) and thus it was a logical choice for this study. Also, VISSIM is currently the only microsimulator that is operated within VISGAOST's optimizations.

\subsection{SSAM}

SSAM is a software application, sponsored by FHWA, which provides safety analysis for simulation-based comparative studies (Gettman et al., 2008). The software processes vehicle trajectory data exported from microscopic traffic simulation models to estimate the frequency and severity of various types of conflicts. By definition, a conflict represents an observable situation in which two or more motor vehicles approach each other in time and space to such an extent that there is risk of collision if their movements remain unchanged (Amundsen and Hyden, 1977).

To determine if a vehicle-to-vehicle interaction is classified as a conflict, the threshold values for two surrogate measures of safety are applied: Time-To-Collision (TTC) and PostEncroachment Time (PET). When TTC $=0$ and/or PET $=0$, SSAM identifies these conditions as if a crash has occurred. If $0<\mathrm{TTC} \leq 1.5 \mathrm{~s}$, and PET $\leq 5.0 \mathrm{~s}$, then SSAM marks the event as a conflict. The 1.5-s TTC threshold and 5.0-s PET threshold are defaults in the SSAM software and they have been used as thresholds in this study too (as recommended by the literature and testing during previous studies, see Gettman et al., 2003, Gettman et al., 2008, and Sabra et al., 2010). For each conflict, SSAM computes a number of corresponding surrogate measures of safety (TTC, PET, etc.) along with their summaries (mean, max, and variance).

SSAM identifies four types of conflicts: crossing (angle), lane-changing, rear-end and unclassified. The type is determined based on the conflict angle, and link and lane information. A 
conflict angle for a pair of vehicles is calculated based on the angle at which these vehicles converge to a hypothetical collision point. In this study we used default SSAM thresholds (Gettman et al., 2008) for conflict angles for various conflict types: the type is classified as a rear-end conflict if the conflict angle $<30^{\circ}$, a crossing conflict if conflict angle $>80^{\circ}$, or otherwise a lane-change conflict.

The SSAM approach was validated using a database of 83 four-legged urban signalized intersections (Gettman et al., 2008). The intersections were modeled in VISSIM and safety was assessed through SSAM. The research included theoretical validation, field validation and sensitivity analysis. The assessments through SSAM showed accuracy similar to that of traditional theoretical crash-prediction equations which are based on average daily traffic volumes.

It should be noted here that some of the driving behavior models, e.g. representing movements of vehicles inside signalized intersections and reaction to signal operations, may still not be good enough to realistically represent vehicular behavior observed in field (Archer, 2004). Thus, an approach where SSAM (or another similar tool) is used to estimate conflicts based on vehicular trajectories pre-processed by microsimulation tools still needs to be validated in the field. However, this approach exhibited significant promise to evaluate traffic engineering alternatives without expensive field crash studies (Gettman et al., 2008); and it will get further validity as driving behavior models within microsimulation tools become more sophisticated.

\subsection{CMEM}

The CMEM is a physically-based, power-demand model developed by University of California at Riverside, the University of Michigan, and Lawrence Berkeley National Laboratory (Scora et al., 2006). After a variety of enhancements, the latest version (3.0) includes sub-models for Light-Duty Vehicles (LDV) and Heavy-Duty Diesel (HDD) vehicles. These sub-models estimate vehicle tailpipe emissions (Carbon monoxide, $\mathrm{CO}$; Hydrocarbons, $\mathrm{HC}$; Nitrogen oxides, $\mathrm{NO}_{\mathrm{x}}$; and Carbon dioxide, $\mathrm{CO}_{2}$ ) in different modes of vehicle operation, e.g., idle, cruise, acceleration, and deceleration. Scora et al. (2006) suggested that temporal and vehicular aggregations were necessary in practice because CMEM was developed to predict emissions for vehicle categories (Scora et al., 2006). The temporal scale ranges from second-by-second, several seconds (mode) to driving cycle or scenario, and the vehicular scale ranges from a specific vehicle, vehicle/technology category, to general vehicle mix or fleet.

CMEM model inputs include traffic composition, vehicle and operation variables (e.g., speed, acceleration, and road grade), and model calibrated parameters (e.g., cold start coefficients and an engine friction factor) (Scora et al., 2006). Outputs are tailpipe emissions and fuel consumption. Emissions $(\mathrm{g} / \mathrm{s})$ are predicted as the product of fuel rate $(\mathrm{FR}, \mathrm{g} / \mathrm{s})$, engine-out emission indices (grams of emission/grams of fuel), and time-dependent Catalyst Pass Fraction (CPF), defined as the ratio of tailpipe to engine-out emissions. CPF is mainly affected by fuel/air ratio and engine-out emissions.

LDV and HDD models have similar structures (Scora et al., 2006). Both are composed of six modules: engine power demand, engine speed, air/fuel ratio for the LDV model or engine control unit for the HDD model, fuel rate, engine-out emissions, and CPF for the LDV model or 
after-treatment pass fraction for the HDD model. Key parameters (e.g., vehicle mass, engine size, fuel type) depend on vehicle technology, fuel delivery system, emission control technology, vehicle age, and other factors. CMEM has been calibrated using data from the National Cooperative Highway Research Program, which includes both engine-out and tailpipe emissions of $\mathrm{CO}, \mathrm{HC}, \mathrm{NO}_{x}$, and $\mathrm{CO}_{2}$ for over 400 vehicles in 36 vehicle/technology categories.

\subsection{VISGAOST}

VISGAOST is an optimization program that optimizes signal timings of traffic controllers based on their performance in VISSIM microscopic simulation. VISGAOST bases its optimization on the stochastic nature of Genetic Algorithms (GAs). The general structure of VISGAOST GA optimization is well documented (Stevanovic et al., 2008). The basic version of VISGAOST is written in C++ and relies on VISSIM's input and output files (PTV, 2010). The key part of the VISGAOST program is a simple GA similar to other GAs used for signal timing optimization (Goldberg, D.E., 1989).

The first version of VISGAOST enabled the optimization of all four basic signal settings, i.e. cycle, offset, splits and phase sequence. Results from the first tests and evaluations confirmed that VISGAOST could generate better timings for VISSIM than those that are running in the field (Stevanovic et al., 2007) or generated by the traditional optimization tools like SYNCHRO.

VISGAOST application was further extended to enable optimization of transit signal priority settings (Stevanovic et al., 2008), signal timings to minimize fuel consumption and vehicular emissions estimated by Comprehensive Modal Emission Model (CMEM) (Stevanovic et al., 2009), among others.

\subsection{VISSIM-SSAM-CMEM-VISGAOST Integration}

Figure 1 shows the integration of VISSIM, SSAM, CMEM, and VISGAOST. A VISGAOST's version, previously used for similar optimizations, was modified to accommodate interfaces between both SSAM and CMEM. The process starts with an initial population of signal timings, generated randomly by VISGAOST based on the existing set of signal timings from field. Each generated signal timing plan is evaluated in VISSIM, resulting in:

- Network-wide performance measures, reflecting mobility aspects of traffic operations, which are directly returned to VISGAOST for further processing.

- Trajectory data file (*.trj), for each vehicle, is transferred to SSAM to compute frequency, severity and types of vehicular conflicts (Gettman et al., 2008), which are then further used in the optimization process in lieu of the real safety metrics.

- Vehicle record files, second-by-second data for each vehicle in the network, which are post-processed in CMEM to generate total fuel consumption which is used in the subsequent optimization steps.

\section{Insert Figure 1}


As a result of the evaluation process VISSIM also outputs a vehicle record file with relevant for the entire simulation period, which is further processed by the VISSIM-CMEM interface and sent to CMEM. CMEM estimates emissions and fuel consumed during the evaluation of that particular signal timing plan. The CMEM estimates are then summed for all vehicles in the network during entire simulation period. VISGAOST receives the summed fuel consumption (or vehicular emission) for each signal timing plan from current population. This value is then used together with mobility and safety metrics for the same signal timing plan to develop a solution in the 3-D optimization space.

A program built in Java connects VISSIM with the LDV and HDD models in CMEM (Figure 1). The program's logic is similar of that described previously (Stathopoulos and Noland, 2003; Chevallier, 2005). For each vehicle VISSIM provides simulation time, a vehicle identifier, a vehicle type (light-duty vehicle or truck), speed, and acceleration/deceleration on a second-bysecond basis. CMEM uses individual vehicle data to estimate instantaneous emissions for each vehicle. Each VISSIM vehicle type is assigned to a CMEM vehicle category.

The assignment of vehicle categories follows a mapping process described in previous studies (Stevanovic et al., 2009), with an assumption that the entire simulated vehicle fleet is composed of Light-Duty Gasoline Vehicles (LDGV) and Heavy-Duty Diesel Vehicles (HDDV) defined in MOBILE 6.2 (EPA, 2003). For other details of emission categories of the vehicles readers are referred to previous studies (Scora et al., 2006). Many vehicle types can be defined in VISSIM but the authors used only passenger cars and heavy vehicles (trucks). Depending on the VISSIM vehicle type the interface program utilizes either the CMEM LDV model (cars) or the CMEM HDD model (trucks). A CMEM model (LDV or HDD) computes fuel consumption and vehicular emissions for each vehicle in the simulation outputs, while the interface program summarizes individual vehicle's fuel consumption and emissions $\left(\mathrm{CO}, \mathrm{HC}, \mathrm{NO}_{\mathrm{x}}\right.$, and $\left.\mathrm{CO}_{2}\right)$ to obtain the total values for the entire road network.

\section{OPTIMIZING SIGNAL TIMINGS TO REDUCE SURROGATE MEASURES OF SAFETY}

When optimizing signal timings with single-objective function, where the goal is to minimize some of the externalities (e.g. fuel consumption and number of conflicts) it is necessary to combine these externalities with mobility metrics (e.g. delay, stops, and throughput) to get meaningful objective functions. Unlike delay, stops and throughput, metrics such as fuel consumed or total number of conflicts are not directly proportional to the efficiency of traffic. For example, less fuel is consumed by standing traffic than by traffic flowing at a speed of 45 mph. Similarly, the number of conflicts in jammed traffic conditions is minimal because vehicles cannot move freely. An attempt to minimize these externalities as sole objective functions would inevitably lead to signal timing solutions that would (e.g. by blocking some traffic movements) decrease overall traffic efficiency. In order to get meaningful results from the optimization process, these externalities are often combined with mobility measures that are directly proportional to traffic activity. For this reason fuel consumption and crash rates (as examples of such externalities) are usually expressed per mile of travel. 
However, for the purpose of optimizations conducted in this study the authors did not have to use any of such combinations of mobility and externality performance metrics. Pareto fronts and multi-objective frameworks, allow for each objective function criteria to be used in its purest/rawest form because each criteria is treated as a separate dimension of the multidimensional search space. Thus, as a mobility measure the authors selected in this study is vehicular throughput - a metric that is less correlated to the environmental and safety externalities than some of the other mobility measures (e.g. correlation between stops and delay and fuel consumption). As a measure of environmental friendliness of a signal timing plan, the authors used fuel consumption (expressed as weight of consumed fuel in grams or kilograms), which is directly correlated to relevant emissions. Finally, total number of vehicular conflicts from SSAM was used to represent safety of a particular signal timing plan. There is a need to further investigate which mobility, safety, and environmental performance measures are the most meaningful for these type of optimizations. However, the authors concentrate here on laying fundamental framework for a 3-dimensional optimization of signal timing plans and they use performance metrics which are either most intuitive, easiest to obtain from the available tools, or recommended by previous studies. Further research should investigate how various performance metrics, used as mobility, safety and environmental criteria, should be used in similar multicriteria optimizations of signal timings.

\subsection{Generalized Approach to 3-Dimensional Optimization in VISGAOST}

Multi-objective optimization is defined as a process where several (usually two), possibly conflicting, objectives are satisfied. In mathematical terms, multi-objective optimization can be defined as a vector of decision variables which satisfies constraints and optimizes a vector function whose elements represent objective functions. These functions form a mathematical description of performance criteria that are usually in conflict with each other. Hence, the term "optimize" means finding a solution with values of all objective functions being acceptable to the decision maker (Osyczka, 1985).

With multiple objective functions, the notion of 'optimum' changes because it is important to find good compromises, or trade-offs, rather than a single solution. The general notion of a multi-objective optimum was introduced by Vilfredo Pareto (Pareto, 1896) with its mathematical formulation given as follows:

A vector of decision variables $\vec{x}^{*} \in F$ is Pareto optimal if there does not exist another

$\vec{x} \in F$ such that $f_{i}(\vec{x}) \leq f_{i}\left(\vec{x}^{*}\right)$ for all $i=1, \ldots, k$ and $f_{i}(\vec{x})<f_{i}\left(\vec{x}^{*}\right)$ for at least one $j$.

Where $F$ denotes the feasible region of the problem.

A Pareto optimal set represents solutions which correspond to vectors $\vec{x}^{*}$, which are called nondominated solutions. Mathematically, Pareto dominance can be described as:

A vector $\vec{u}=\left(u_{1}, \ldots, u_{k}\right)$ is said to dominate $\vec{v}=\left(v_{1}, \ldots, v_{k}\right)$ (denoted by $\vec{u} \preceq \vec{v}$ ) if and only if $u$ is partially less than $v$, i.e., $\forall i \in\{1, \ldots, k\}, u_{i} \leq v_{i} \wedge \exists i \in\{1, \ldots, k\}: u_{i}<v_{i}$. 
By definition, solution A dominates solution B if solution A is no worse in all objectives than solution B and provides an improvement in at least one objective. Considering this definition, the best solutions are always ones that are nondominated by other solutions. Pareto Front represents a plot of objective functions whose nondominated vectors are in the Pareto optimal set.

In this research, the multi-objective optimization is implemented and applied to signal settings optimization problems to find timing plans with the best compromises between throughput, number of conflicts, and amount of consumed fuel. The optimal trade-off between the three competing objectives is presented with an approximation of a 3-dimensional Pareto Front. Since the true Pareto Front is unknown for this problem (due to the stochastic nature of evolutionary algorithms based on stochastic traffic simulation), multi-objective optimization aims to approximate this set.

Details of GA procedure deployed within VISGAOST are presented elsewhere (Stevanovic et al., 2007 and Stevanovic et al., 2013a). Here, authors focus on changes introduced into the previous GA structure to enable 3-dimensional optimization within VISGAOST. The main differences are related to selection and the elitism operations within GA. The new selection favors "fitter" solutions for reproduction, but the fitness is determined based on the nondominated ranks of competing objectives, as opposed to being based on a single-objective value. A process of ranking solutions starts when the set of nondominated individuals/solutions in the population is assigned a rank of 1 . This set represents a Pareto Front for the current population. Then the non-dominated individuals in the remaining set of solutions are assigned a domination rank of 2 , etc. The basic steps required to rank a whole population $p$ are given below. One should note that the rank of all timing plans that are members of a nondominated front $f^{r}$, is $r$. Obviously, the nondominated front $f^{1}$ of a population represents the Pareto Front of that population.

\section{Algorithm for Ranking Solutions \& Creating Pareto Fronts}

\section{Step 0:Initializing}

$p$, population of timing plans

$r$, rank of current non-dominated front

$f^{r}$,current non-dominated front

$r=1$

Step 1:Testing Termination Criterion

IF $(p=\varnothing)$

$$
\begin{gathered}
\text { Stop and Terminate the Search } \\
f^{r}=\left\{t p^{1}\right\}, t p^{1} \in p \\
\text { GO TO Step } 2
\end{gathered}
$$

Step 2: Searching for Non-dominated Front

$$
\begin{aligned}
& \text { FOR } \forall t p^{k}\left(t p^{k} \in p \wedge t p^{k} \notin f^{r}\right) \\
& f^{r}=f^{r} \cup\left\{t p^{k}\right\} \\
& \text { FOR } \forall t p^{m}\left(t p^{m} \in f^{r} \wedge t p^{m} \neq t p^{k}\right) \\
& \operatorname{IF} \quad\left(\left(\left(\operatorname{conflicts}\left(t p^{k}\right)<\operatorname{conflicts}\left(t p^{m}\right)\right) \wedge\right.\right.
\end{aligned}
$$




\section{Step 3:Reinitializing}

$$
\begin{gathered}
\quad\left(\text { throughput }\left(t p^{m}\right)<\operatorname{throughput}\left(t p^{k}\right)\right) \wedge \\
\left.\left(\text { fuel }\left(t p^{k}\right)<\text { fuel }\left(t p^{m}\right)\right)\right) \\
\text { THEN } f^{r}=f^{r} \backslash\left\{t p^{m}\right\} \\
\text { ELSE IF }\left(\left(\left(\operatorname{conflicts}\left(t p^{m}\right)<\operatorname{conflicts}\left(t p^{k}\right)\right) \wedge\right.\right. \\
\quad\left(\text { throughput }\left(t p^{k}\right)<\operatorname{throughput}\left(t p^{m}\right)\right) \wedge \\
\left.\quad\left(\text { fuel }\left(t p^{m}\right)<\text { fuel }\left(t p^{k}\right)\right)\right) \\
\text { THEN } f^{r}=f^{r} \backslash\left\{t p^{k}\right\}
\end{gathered}
$$

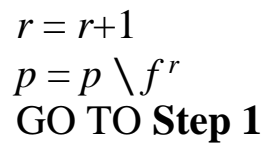

In the next step, the binary tournament selection is conducted during which $\mathrm{N}$ (population) pairs of timing plans are randomly selected. The timing plans with the lower domination ranks are chosen to contribute to the next generation. If both selected timing plans have the same rank, the final choice is determined randomly. The binary tournament selection is repeated until a mating pool, of the same size as the population, is obtained. Further creation of a new population using the four basic GA operations is the same as described for single-objective optimization (Stevanovic et al., 2008).

Once the child population is created, it is combined with the parent population and the domination ranks are reassigned. Finally, the elitist replacement scheme finds the $\mathrm{N}$ best individuals within the united population and keeps them for future generation. However, the selection process of $\mathrm{N}$ best individuals is not necessarily trivial. If the size of the nondominated front $f^{r}$ is smaller than $\mathrm{N}$, all of the nondominated timing plans become members of the new population. The remaining members are chosen from subsequent nondominated fronts in order of their ranking until the new population is complete. Conversely, if the size of the nondominated front $f^{r}$ is higher than $\mathrm{N}, \mathrm{N}$ timing plans for the new population are chosen randomly (Deb et al., 2002). The basic operations in the multiple-objective optimization process performed by VISGAOST are summarized below. The process also includes procedures to read/write signal timings from/to Ring-Barrier Controllers (RBC) in VISSIM, which has been covered in previous studies (Stevanovic et al., 2008).

\section{Generalized Genetic Algorithm (VISGAOST) with Pareto 3-D Optimization}

Step 0:Initializing

$G$, total number of generations

$T$, total number of timing plans per generation

$\varepsilon$, convergence threshold

$i$, current number of population

$p f$, Pareto Front

$i=0$

$p f=\varnothing$

Generation of initial population $p^{i}$ of timing plans $t p^{k}, \forall k \in[1, . . \mathrm{T}]$ 
- read field timing plan $t p^{l}$ from RBC database

- generate $t p^{k}, \forall k \in[2, . . \mathrm{T}]$

- rank population $p^{i}$

- define $p f=$ paretoFront $\left(p^{i}\right)$

Step 1:Evaluating Population

Evaluation of $t p^{k} \in p^{i}, \forall k \in[1, . . \mathrm{T}]$

- write $t p^{k}$ to RBC database

- simulate (and evaluate network performance of) $t p^{k}$

- estimate safety measures (through SSAM) for $t p^{k}$

- estimate fuel consumption/emissions measures (through CMEM) for $t p^{k}$

Step 2:Testing Termination Criterion

IF ( $\mathrm{i}=\mathrm{G})$

ELSE

Stop and RETURN $p f$

GO TO Step 3

Step 3: Generating New Population

$i=i+1$

Generation of new population $p^{i}$

- generate $p^{i}$ through GA-operations

- form combined population $p^{u}=\left(p^{i-1} \cup p^{i}\right)$

- rank population $p^{u}$

- define $p^{i}=\left(\mathrm{T}\right.$ best timing plans from $\left.p^{u}\right)$

- define $p f=\operatorname{paretoFront}\left(\operatorname{paretoFront}\left(p^{u}\right) \cup p f\right)$

GO TO Step 1

\subsection{Optimization with No Constraints - A Theoretical Approach}

When defining the search process within optimization framework, one can opt for a comprehensive coverage of the search space or for efficiency of reaching an optimal solution. Various literature on stochastic optimization and evolutionary algorithms cover these subjects, which also show how various GA operators can be used to apply one of these two approaches. Here we discuss how these two approaches (opting for "coverage" or "efficiency") impact experiments in this study. The "coverage" approach is where the authors set the optimization process to explore entire (or as much as possible) search space, with minimal constraints (other than those embedded in the NSGA-II structure). As such, this approach represents a theoretical approach, where many solutions, which would not be practically acceptable in the real-world signal operations, are generated and evaluated.

The randomization of the created solutions, which increases coverage of the search space, is achieved by modifying the algorithm (pseudo code presented above) by giving less weight to the best signal timings from previous generation in the selection process of the signal timings 
which will be combined for reproduction. This modification increases randomness of the selected timing plans and thus increases chances that plans from the entire domain are covered. The pseudo code of this modification in the plan selection can be expressed as:

$t p^{l}$, randomly selected $t p$ from current population of timing plans

$t p^{2}$, randomly selected $t p$ from current population of timing plans

$r n d$, randomly generated number within interval $[0,1]$

IF $(r n d<0.5)$

THEN

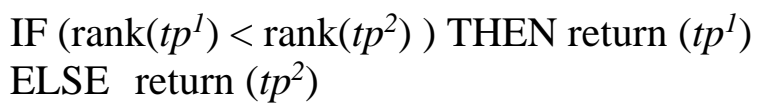

\subsection{Constrained Optimization - A Practical Approach}

The second optimization approach deals with more of practical concerns (hence the name), understanding that not many signal timing professionals, would opt to reduce mobility (throughput) for a signal timing plan that may (or may not) decrease safety concerns and environmental impacts. The logic behind this reasoning is following: while serving few extra vehicles may have significant impact on traffic performance in urban (e.g. oversaturated) conditions, the correlation between conflicts and fuel consumption, as proxies of crashes and air pollution, respectively, may not be so obvious. For example, estimated conflict(s) under particular conditions may result in a crash, while in some other conditions there will not be any crashes. Crashes are highly random events that primarily depend on individual behavior of the driver(s), which is not easy to model or correlate with the number of vehicular conflicts. Similarly, for the same amount of fuel consumed in a certain traffic network other factors (such as temperature, air pressure, and wind) may significantly impact whether air pollution is significant or not. This uncertainty in correlation between estimated and measurable externality metrics, weakens the argument that the optimization process should select solutions that deteriorate mobility in order to improve externalities. To account for this view the authors considered an approach where mobility is given a threshold below which solutions are not likely to be selected (even if they improve safety and environmental factors). The following text describes the algorithm for solution ranking and creation of Pareto fronts is modified to implement the constraint in throughout reduction.

The line "Stop and Terminate the Search" in the original algorithm was replaced with the lines:

FOR $\forall t p^{k}\left(t p^{k} \in p\right)$

IF throughput $\left(t p^{k}\right)<\frac{98 * T_{\text {initial }}}{100}$

$\mathrm{THEN} \operatorname{rank}\left(t p^{k}\right)=99$ 
This code modification ensured that the rank value of unsuitable signal timing plan (e.g. with a throughout 2 or more percent below the nominal value) is significantly increased (e.g. from 1 to 5), leading to the significant decrease in chances of being selected for reproduction.

The other concern of practical GA optimization is an ability to quickly converge to the optimal solution. Thus GA operators can be set in such a way that better solutions are given more chances of getting reselected in future generation, which streamlines the process of creating 3dimensional surfaces in certain direction. Particular changes to the original code, where among two randomly selected timing plans, the better plan is selected for reproduction, is explained with the following pseudo code:

$t p^{l}$, randomly selected $t p$ from current population of timing plans

$t p^{2}$, randomly selected $t p$ from current population of timing plans

IF $\left(\operatorname{rank}\left(t p^{l}\right)<\operatorname{rank}\left(t p^{2}\right)\right)$ THEN return $\left(t p^{l}\right)$

ELSE return $\left(t p^{2}\right)$

\section{EXPERIMENTS}

Experiments conducted in this study were divided into two major parts, following reasoning described in sections 4.2 and 4.3. In both parts, a real-world network is used as a case study to demonstrate feasibility of the approach and investigate performance of the three objective functions. In the first part (results presented in section 5.3) the authors took a theoretical approach and allowed optimization to search widely for solutions without forcing Pareto Front convergence and without any practical constraints. In the second part (results presented in section 5.4), previously described approach, which forces a quicker Pareto front convergence, was applied together with practical limitations of possible throughput reduction. The second part of the experiments was also used to evaluate performance and quality of signal timing plans obtained through single-objective optimizations, and other methods, from the previous studies.

\subsection{Case-Study Network}

A 1.5-mile section (between 4000 and 5200 West) of 3500 South Street (SR 171), located in West Valley City, UT was used as a test bed for optimization experiments. This section of 3500 South, shown in Figure 2, has 5 actuated-coordinated signalized intersections and represents one of the major E-W arterials in the Salt Lake County. Levels of service and basic geometry are given for all intersections in Figure 2. This particular network was selected for this research because it is large enough to provide test-bed of realistic coordinated operations in the field and at the same time small enough to execute computationally-expensive optimization experiments.

PM peak traffic volumes and turning-movement counts were collected for most of the turning movements in the network. A few missing traffic counts were estimated in the flowbalancing spreadsheets. Field travel times were collected by floating-car technique with GPS units. All of the data were collected during the PM peak period on Tuesdays, Wednesdays and Thursdays under fair weather and dry pavement conditions, during a busy traffic season.

A VISSIM model of the study case segment was built, calibrated and validated based on data from the field. To calibrate the VISSIM model timings from the field were used. These included speed limits, PM peak 15-minute turning movement counts, and queue lengths at some 
intersections. To validate the model, travel times (floating car with GPS) along the arterial were measured, while recording passing times at each intersection. Figure 3 shows the results of the calibration and validation efforts.

\section{Insert Figure 2}

\section{Insert Figure 3}

\subsection{VISGAOST Optimizations}

Two aforementioned optimization experiments were conducted; all starting from the same base field signal timings. Each optimization was based on evaluations of throughput (number of vehicles that completed their trips, from VISSIM's network performance files), total number of conflicts (as surrogate measures of safety from SSAM), and total fuel consumption (from CMEM). These metrics represented values accumulated during 30 minutes of simulation time, with an additional 15 minutes of warm up time. Each optimization had 2000 evaluations of various signal timing plans - 20 signal timing plans were operated through GA procedures for each of 100 generations. All VISSIM evaluations within VISGAOST optimizations were executed for a single random seed (for the purpose of computing efficiency). The GA parameters used were default VISGAOST parameters that were justified in previous studies (Stevanovic et al., 2007). In total, optimization experiments took several weeks of continuous run time on a personal computer.

\subsection{Optimization Results - A Theoretical Approach}

Figure 4 shows results of the non-constrained optimization experiments. Each of the four charts in Figure 4 shows signal timing solutions in the search space, as the optimization evolved through the 100 generations (2000 evaluations). Part A) of Figure 4 shows the signal timing solutions in 3-dimensional space, with various markers representing solutions which are members of the three samples of Pareto fronts from the $5^{\text {th }}, 50^{\text {th }}$, and $100^{\text {th }}$ (final) generation. The series of these three Pareto fronts is supposed to show (in Parts B), C), and D) of Figure 4) how these fronts progress towards 'optimal region' in the 3-dimensional space of mobility, safety, and environment. Such optimal region is clearly located in the upper front corner of the imaginary cube in Figure 4, Part A) where throughputs are high, and fuel consumptions and number of conflicts are low.

Parts B), C), and D) of Figure 4 show signal timing solutions in 2-dimensional spaces of throughput and conflicts, throughput and fuel consumption, and fuel consumption and conflicts, respectively. All three 2-dimensional charts show that the search space has been significantly (although not fully) covered by evaluated solutions and that the optimization 'pushed' solutions (through intelligent selection, a feature of GAs) towards the 'optimal region'.

\section{Insert Figure 4}


More details of how Pareto fronts progress towards optimality region are shown in Figure 5 (especially Parts B), C), and D)), which are the close-up versions of the same charts in Figure 4. Only difference in Figure 5 is that some of the solutions in outer search space are sacrificed for the clarity of the views. In Figure 5, the three Pareto fronts (for generations 5, 50, and 100) are colored and displayed as 2-dimensional surfaces. One should note that when a 3-dimensional Pareto front is shown in any of the 2-dimensional plots, such fronts are usually not approximated by convex/ concave lines as in common 2-dimensinal cases, but by closed areas, as shown in Figure 5. The optimization results in Figure 5 clearly show that the Pareto fronts: 1 . Move in the right direction (towards the 'optimality region'), and 2. Gain more solution-members, as the optimization progresses.

\section{Insert Figure 5}

\subsection{Optimization Results - A Practical Approach}

Figure 6 shows optimization results, which are equivalent to the results shown in Figure 4 but only for the constrained optimization search, where throughput was not given flexibility to vary as much as in the previous case, and where the Pareto front propagation process was more streamlined. One can easily see in Figure 6 (all charts) that the solutions are much denser in the 'optimality region' and search space is much less covered by the solutions than in Figure 4. In addition to regular Pareto front points from four generations $\left(6^{\text {th }}, 23^{\text {rd }}, 53^{\text {rd }}\right.$, and $\left.100^{\text {th }}\right)$ four other points (representing "signal timing scenarios") are shown in Figure 6. While performance of these signal timing scenarios will be discussed later, it is important to note that they represent outputs of the study where Green Light Speed Advisory Systems (GLOSA) are compared to commonly optimized signal timings (Stevanovic et al., 2013b). GLOSA assumes drivers' participation (by accepting advisory speeds) in signalized operations, where reduced/increased traffic flow speeds reduce stops and unnecessary delay at the intersections. Details of GLOSA performance and its comparisons with the other scenarios are presented elsewhere (Stevanovic et al., 2014) but here the authors summarize features of those four signal timing scenarios (named as presented in the legends of Figure 6):

- FixedOpti - field signal timings (actuated) converted into their fixed-time counterparts and optimized to minimize the PI (single-objective function weights of delay and stops)

- Glosa-FixedOpti - GLOSA applied to the FixedOpti scenario

- ActuatedOpti - field signal timings (actuated-coordinated) optimized to minimize the PI

- Glosa-ActuatedOpti - GLOSA applied to the ActuatedOpti scenario

All of these additional signal timing scenarios were executed on the same network, under the same operational conditions (30 minutes, warm-up time, random seed, etc.), and their outcomes were post-processed in SSAM and CMEM consistently to the optimization runs.

\section{Insert Figure 6}


Figure 7 shows a closer look into Pareto fronts developed under constrained optimizations and the four signal timing scenarios from the previous studies. Regarding the Pareto fronts, similar behavior has been observed (consistent progress towards higher throughputs and lower fuel consumptions and number of conflicts) as from the experiments with unconstrained optimizations. One should note from comparisons of Figures 5 and 7, that the Pareto fronts in the non-constrained optimizations (Figure 5) have been able to find few solutions which generate lower fuel consumptions and number of conflicts (while the throughputs are about the same) than the Pareto fronts from Figure 7 (constrained optimizations). This result is expected as it is probably a consequence of a broader search that is performed under nonconstrained optimization leading to few better solutions, from the externality standpoint.

Regarding the four signal timing scenarios from previous studies, some of them are quite well placed in comparison to the achieved Pareto fronts. For example, optimal plans for actuatedcoordinated system (encircled X sign) is quite better in fuel consumption than any of the other solutions. This should not be a big surprise considering that the PI is a surrogate for fuel consumption and the fact that PI in this previous optimization was evaluated through 400 additional signal timing plans.

It should be also noted that all of the four signal timing scenarios outperformed field signal timing plans by reducing travel times up to $10 \%$, fuel consumption by up to $12.5 \%$, and number of stops by up to $75 \%$ (GLOSA). Considering the proximity of these four points to the Pareto fronts in Figure 7, one would expect similar improvements (over the field signal timings) of the signal timing plans constituting final 3-dimensional Pareto fronts developed in this study.

\section{Insert Figure 7}

Original finding of this study is that a scenario where the GLOSA is applied with optimized fixed-time plans yielded the lowest number of vehicular conflicts. This finding is very original since there were no previous studies that evaluated performance of GLOSA, or a similar Connected Vehicle technology, on the total number of vehicular conflicts. This finding in not unexpected since the GLOSA does provide smoother flows at the signalized intersections, which is supposed to help reduce number of potential conflicts. The other scenarios (GLOSA with optimal actuated-coordinated plans or fixed-time optimal plans) did not prove to be solutions which would improve on the Pareto fronts obtained through the 3-dimensional optimizations.

\subsection{Significance of the Optimization Results}

Table 1 shows combined Pareto front solutions (signal timings) from both constrained and notconstrained optimizations. Shaded rows represent solutions from not-constrained optimizations and clearly those solutions favor externality metrics more than they favor the throughput. Oppositely but consistently, the optimizations with constrained throughputs produced slightly better throughputs but at the expense of externalities.

\section{Insert Table 1}


The last row in the Table 1 shows the differences (in percent) between lowest and highest value for each of the criteria. It is clear that these differences are not large for throughputs and for fuel consumption (up to $3 \%$ and up to $4.5 \%$, respectively). The highest difference is (and considerably high) for number of vehicular conflicts but this criterion is also the least accurately (comparing to the other two) estimated based on the simulation outputs. If/when these results are exposed to stochastic nature of traffic flows (which will be done in future experiments), it is reasonable to expect that the solution within a Pareto front will not be so different to recognize any significant practical difference from their application. It will be way more important to perform optimizations in which original/field signal timings (also part of the early Pareto fronts) are pushed through the optimization process closer to the 'optimality region'.

Figure 8 shows practical differences of the signal timing plans extracted from the two Pareto front optimizations. The best signal timings for externalities (these are plans 1-8 from the Table 1, they almost coincide as the best plans both for fuel consumption and conflicts) were averaged in a meaningful way to create a 'representative' signal timing plan for the externalities. Similarly, the best signal timing plans for throughput (e.g. 13-21) were averaged to find the representative signal timing plan for the throughput. The resulting signal timings are presented in Figure 8. One can observe that the cycle length (it is the same for all intersections) for externality representative timing plan $(127 \mathrm{sec})$ is slightly higher than the cycle length for the throughput representative timing plan $(112 \mathrm{sec})$. On the other hand, most of the splits are very similar while the offsets are mostly quite different. Considering that offsets work in cyclical way (multiple offsets can work well for the same cycle length and distance between intersections), further analysis is required to investigate how meaningful proposed offset changes are. Regarding the green splits, it is quite clear that these do not change much for either representative timing plan. Few cases where the splits (for the same phases) are significantly different could be anomalies of the evolutionary algorithm (e.g. not enough plans were investigated) rather than a meaningful result.

\section{Insert Figure 8}

\section{CONCLUSIONS}

The objective of this study was to present a new method of integrating traffic simulation, surrogate safety assessment, fuel consumption and emission modeling, and signal timing optimization tool to optimize signal timings in such a way to achieve a 3-dimensional surface of signal timing plans that could help operators make smart decisions when trading off between mobility, safety, and environment. The case study used a network of 5 signalized intersections in West Valley City, Utah, as a test bed. For this arterial, two multi-criteria optimizations were completed to determine a balance between mobility, safety and environment. In addition, a set of previous developed signal timing scenarios, including some of the Connected Vehicle technologies such as GLOSA, were conducted to evaluate the quality of the 3-dimensional Pareto front solutions. Based on the described integration processes and observed findings, it can be concluded that: 
- The process of developing 3-dimensioanl surfaces of signal timing plans that provide tradeoff between mobility, safety, and environment is a feasible endeavor. This process lead to proper Pareto front improvements, which in given cases lead to solutions which significantly improved signal timings, in some criteria more than single-objective optimizations.

- The optimal balance between mobility, safety and environmental impacts does not seem to produce signal timings which are very different from each other. Differences between various criteria of different signal timing plans on the 3-dimensional Pareto front surface do not seem to be large; mainly less than $5 \%$ with up to $35 \%$ for the safety/number of conflicts. Future studies should test the hypothesis that such differences may get more or less pronounced when tested in the stochastic traffic flows.

- The optimal signal timing plans, performed through common single-objective-function optimizations, seem to deliver signal timings which are comparable to the ones from the 3-dimensional Pareto fronts. Optimal signal timings for PI in actuated-coordinated system show that such plans are the best to minimize fuel consumption. This results may be a consequence of the fact that optimization for PI had $20 \%$ more iterations than the Pareto front optimization; hence, higher a chance to reach the optimal solution.

- GLOSA operations (for fixed but optimized signals) seem to perform surprisingly well from the safety standpoint, reducing the number of vehicular conflict to the levels that could not be achieved with any signal optimization. Originally developed to reduce fuel consumption it seems that this speed advisory combination with signal timings has promising future for the improvement of safety on arterial streets. Further experiments are needed to confirm these results.

- When representative signal timings are developed for externalities (safety and fuel consumption) on one side and mobility (throughput) on the other, the differences in signal timings are the least significant among the splits. Cycle lengths seem to be close but significant enough, whereas the offsets are considerably different. Experiments described in this paper do not delve in the details of how certain signal timings affected local/intersection optimization criteria but this remains a goal for some of the future studies.

More research is also needed to test this approach on a variety of networks and traffic conditions and to potentially find a good composite measure (of mobility, safety, and environment) that would enable good quality single-objective optimizations. From this perspective it seems that the traditional PI might be a good starting point for such a measure but further research is needed to both integrate (surrogate) safety and calibrate such a measure. Also, considering that certain conflicts (e.g. crossing) may have more severe impacts than the others (e.g. rear-end) it is needed to investigate use of a "safety (performance) index" which would use various 'severity weights' assigned to various conflict types. Following, the field would benefit from integrating stochastic processes to represent variety of traffic conditions in the optimization process; this would lead to more robust signal timings. Finally, the next generation of traffic controllers and control policies is likely to use vehicle to vehicle and vehicle to infrastructure communication. It is necessary to investigate how this type of control will facilitate the optimization of various externalities. 


\section{ACKNOWLEDGEMENTS}

The research presented here is partially funded by National Science Foundation Grant \# 1229616 "MRI: Development of Instrumentation to Support Multi-Technology Vehicular Networking Systems Research."

The authors dedicate this paper to late Dr. Mathew G. Karlaftis, a great scholar and personal friend. May the God give his soul eternal peace.

\section{REFERENCES}

Abbas, M.M., Sharma, A., Jung, Y.S., 2005. Optimization of time of day plan Scheduling using a multi-objective evolutionary algorithm. In proceedings Transportation Research Board, 84th Annual Meeting, Washington, D.C.

Abbas, M.M., Sharma, A., 2006. Multiobjective plan selection optimization for traffic responsive control. Journal of transportation engineering 132, 376-384.

Abu-Lebdeh, G., Benekohal, R.F., 1997. Development of traffic control and queue management procedures for oversaturated arterials. Transportation Research Record: Journal of the Transportation Research Board 1603, 119-127.

Agrawal, G., Lewis, K., Chugh, K., Huang, C., Parashar, S., Bloebaum, C., 2004. Intuitive visualization of Pareto frontier for multi-objective optimization in n-dimensional performance space. Presented at the 10th AIAA/ISSMO multidisciplinary analysis and optimization conference, pp. 1523-1533.

Amundsen, F., Hyden, C., 1977. Proc. of first workshop on traffic conflicts. Institute of Transport Economics, Oslo, Norway.

Archer, J. and Kosonen, I. 2000. The potential of micro-simulation modeling in relation to traffic safety assessment. Proc. Of the ESS Conference, Hamburg, Germany.

Archer, J. 2004. Methods for the Assessment and Prediction of Traffic Safety at Urban Intersections and their Application in Micro-simulation Modeling. PhD Thesis. Division of Transport and Logistics, Royal Institute of Technology (KTH), Stockholm, Sweden.

Archer, J. and Young, W. 2010. Traffic micro-simulation approach to estimate safety at unsignalized intersections. CD ROM. Presented at 89th Transportation Research Board Annual Meeting, Washington, D.C.

Barth, M., An, F., Younglove, T., Scora, G., Levine, C., Ross, M., Wenzel, T., 2000. Comprehensive modal emission model (CMEM), version 2.0 user's guide. University of California, Riverside. 
Blasco, X., Herrero, J., Sanchis, J., Martínez, M., 2008. A new graphical visualization of $<$ i $>$ ndimensional Pareto front for decision-making in multiobjective optimization. Information Sciences 178, 3908-3924.

Bojanowski, C., Kulak, R.F., 2011. Multi-objective optimisation and sensitivity analysis of a paratransit bus structure for rollover and side impact tests. International Journal of Crashworthiness 16, 665-676.

Branke, J., Goldate, P., Prothmann, H., 2007. Actuated traffic signal optimization using evolutionary algorithms. In Proceedings of the 6th European Congress and Exhibition on Intelligent Transport Systems and Services.

Cevher, V., Kaplan, L., 2008. Pareto frontiers of sensor networks for localization. Presented at the Information Processing in Sensor Networks, 2008. IPSN'08. International Conference on, IEEE, pp. 27-38.

Ceylan, H., Bell, M.G., 2004. Traffic signal timing optimisation based on genetic algorithm approach, including drivers' routing. Transportation Research Part B: Methodological 38, 329_342.

Ceylan, H., 2013. Optimal Design of Signal Controlled Road Networks Using Differential Evolution Optimization Algorithm. Mathematical Problems in Engineering 2013.

Chen, K., Yu, L., 2007. Microscopic traffic-emission simulation and case study for evaluation of traffic control strategies. Journal of Transportation Systems Engineering and Information Technology 7, 93-99.

Chen, O. J., \& Ben-Akiva, M. E., 1998. Game-theoretic formulations of interaction between dynamic traffic control and dynamic traffic assignment.Transportation Research Record: Journal of the Transportation Research Board, 1617(1), 179-188.

Chen, X., Qian, D., Shi, D., 2011. Multi-objective optimization method of signal timing for the non-motorized transport at intersection. Journal of Transportation Systems Engineering and Information Technology 11, 106-111.

Chevallier, E., 2005. Microscopic Modeling Framework for Estimating Emissions from Traffic Management Policies. MS thesis. University of London.

Cohon, J.L., 2010. Multiobjective programming and planning, 1978. Academic, New York. Deb, K., 2001. Multi-objective optimization using evolutionary algorithms. John Wiley \& Sons.

Deb, K., Pratap, A., Agarwal, S., Meyarivan, T., 2002. A fast and elitist multiobjective genetic algorithm: NSGA-II. Evolutionary Computation, IEEE Transactions on 6, 182-197. 
Diakonikolas, I., 2011. Approximation of multiobjective optimization problems. Doctoral dissertation, Columbia University.

Drummond, K., Hoel, L.A., Miller, J.S., 2002. A Simulation-based Approach to Evaluate Impacts of Increased Traffic Signal Density. Research report of Virginia Transportation Research Council, Charlottesville, Virginia.

EPA U.S. 2003. User's Guide to MOBILE6.1 and MOBILE6.2. http://www.epa.gov/otaq/models/mobile6/420r03010.pdf

Ezzat, A.A., Farouk, H.A., El-Kilany, K.S., Moneim, A.F.A., 2014. Optimization Using Simulation of Traffic Light Signal Timings. Proceedings of the 2014 International Conference on Industrial Engineering and Operations Management Bali, Indonesia.

Fikse, K., 2011. Accelerating the Search for Optimal Dynamic Traffic Management. Doctoral dissertation, Master Thesis, University of Twente.

Fonseca, C.M., Fleming, P.J., 1993. Genetic Algorithms for Multiobjective Optimization: FormulationDiscussion and Generalization. Presented at the ICGA, pp. 416-423.

Foy, M.D., Benekohal, R.F., Goldberg, D.E., 1992. Signal timing determination using genetic algorithms. Transportation Research Record. Transportation Research Record 1365, 108-115.

Gartner, N. H., \& Al-Malik, M., 1996. Combined model for signal control and route choice in urban traffic networks. Transportation Research Record: Journal of the Transportation Research Board, 1554(1), 27-35.

Gettman, D., Head, L., 2003. Surrogate Safety Measures from Traffic Simulation Models. Report No. FHWA-RD-03-050, Federal Highway Administration, Washington, DC.

Gettman, D., Pu, L., Sayed, T., Shelby, S., 2008. Surrogate Safety Assessment Model and Validation: Final Report. Report No. FHWA-HRT-08-051, Federal Highway Administration, Washington, DC.

Girianna, M., Benekohal, R.F., 2002. Dynamic signal coordination for networks with oversaturated intersections. Transportation Research Record: Journal of the Transportation Research Board 1811, 122-130.

Girianna, M., Benekohal, R.F., 2004. Using genetic algorithms to design signal coordination for oversaturated networks. Journal of Intelligent Transportation Systems 8, 117-129.

Goldberg, D.E., 1989. Genetic Algorithms in Search, Optimization and Machine Learning. Addison-Wesley- Publishing Co. Inc. Massachusetts. 
Gollub, C., de Vivie-Riedle, R., 2009. Multi-objective genetic algorithm optimization of 2D-and 3D-Pareto fronts for vibrational quantum processes. New Journal of Physics 11, 013019.

Hadi, M.A., Wallace, C.E., 1993. Hybrid genetic algorithm to optimize signal phasing and timing.Transportation research record 1421, 104-112.

Hallmark, S.L., Guensler, R., 1999. Comparison of speed-acceleration profiles from field data with NETSIM output for modal air quality analysis of signalized intersections. Transportation Research Record: Journal of the Transportation Research Board 1664, 40-46.

Holdsworth, C., Kim, M., Liao, J., Phillips, M.H., 2010. A hierarchical evolutionary algorithm for multiobjective optimization in IMRT. Medical physics 37, 4986-4997.

Horn, J., Nafpliotis, N., Goldberg, D.E., 1994. A niched Pareto genetic algorithm for multiobjective optimization. Presented at the Evolutionary Computation, 1994. IEEE World Congress on Computational Intelligence., Proceedings of the First IEEE Conference on, Ieee, pp. 82-87.

Jousselme, A.-L., Maupin, P., Debaque, B., Prevost, D., 2012. A decision support tool for a Ground Air Traffic Control application. Presented at the Information Fusion (FUSION), 2012 15th International Conference on, IEEE, pp. 60-67.

Kesur, K.B., 2010. Generating More Equitable Traffic Signal Timing Plans. Transportation Research Record 2192, 108-115.

Klunder, G., Abdoelbasier, A., Immers, B., 2006. Development of a micro-simulation model to predict road traffic safety on intersections with surrogate safety measures. Paper (No. 1894) presented at ITS World Congress in London, UK.

Knowles, J., Corne, D., 1999. The pareto archived evolution strategy: A new baseline algorithm for pareto multiobjective optimisation. Presented at the Evolutionary Computation, 1999. CEC 99. Proceedings of the 1999 Congress on, IEEE.

Kosonen, I., Ree, S., 2000. The potential for microscopic simulation in traffic safety and conflict studies. In: Proc. of Conference on Road Safety on Three Continents, Pretoria.

Koupal, J., Michaels, H., Cumberworth, M., Bailey, C., Brzezinski, D., 2002. EPA's plan for moves: A comprehensive mobile source emissions model. In Proceedings of the 12th CRC OnRoad Vehicle Emissions Workshop, San Diego, CA.

Kovvali, V., Messer, C., 2002. Sensitivity analysis of genetic algorithm parameters in traffic signal optimization. Presented at the 81st Annual Meeting of the Transportation Research Board, Washington, DC. 
Kwak, J., Park, B., Lee, J., 2012. Evaluating the impacts of urban corridor traffic signal optimization on vehicle emissions and fuel consumption. Transportation Planning and Technology 35, 145-160.

Lertworawanich, P., Kuwahara, M., Miska, M., 2011. A new multiobjective signal optimization for oversaturated networks. Intelligent Transportation Systems, IEEE Transactions on 12, 967976.

Li, H., Guo, H., Zeng, S.X., Tam, V.W., Diao, X., 2011. A pareto multi-objective optimization approach for solving time-cost-quality tradeoff problems. Technological and Economic Development of Economy 22-41.

Li, X., Li, G., Pang, S.-S., Yang, X., Tian, J., 2004. Signal timing of intersections using integrated optimization of traffic quality, emissions and fuel consumption: a note. Transportation Research Part D: Transport and Environment 9, 401-407.

Li, Y., Yu, L., Tao, S., Chen, K., 2013. Multi-Objective Optimization of Traffic Signal Timing for Oversaturated Intersection. Mathematical Problems in Engineering 2013.

Liao, T., Machemehl, R., 1996. Optimal Traffic Signal Strategy for Fuel Consumption and Emissions Control at Signalized Intersections. Presented at the Proceedings of 24th European Transport Forum, Brunel University, England.

Ma, D., Nakamura, H., 2010. Cycle Length Optimization at Isolated Signalized Intersections from the Viewpoint of Emission. Traffic and Transportation Studies 2010, 275-284.

Ma, W., Yang, X., Pu, W., Liu, Y., 2010. Signal timing optimization models for two-stage midblock pedestrian crossing. Transportation Research Record: Journal of the Transportation Research Board 2198, 133-144.

Ma, X., 2012. Multi-criteria evaluation of optimal signal strategies using traffic simulation and evolutionary algorithms. Proceedings of International Congress on Environmental Modelling and Software (iEMSs 2012).

Madetoja, E., Ruotsalainen, H., Monkkonen, V.-M., Hamalainen, J., Deb, K., 2008. Visualizing multi-dimensional Pareto-optimal fronts with a 3D virtual reality system. Proceedings of the International Multiconference on Computer Science and Information Technology, Wisa, Poland, pp. 907-913.

Nam, E.K., Gierczak, C.A., Butler, J.W., 2003. A Comparison of real-world and modeled emissions under conditions of variable driver aggressiveness. Presented at the 82nd Annual Meeting of the Transportation Research Board, Washington, DC. 
Oda, T., Kuwahara, M., Niikura, S., 2004. Traffic signal control for reducing vehicle carbon dioxide emissions on an urban road network. Presented at the Proceedings of 11th World Congress on ITS (CD-ROM).

Osyczka, A. 1985. Multicriteria optimization for engineering design. Design optimization, 1 (1985), 193-227.

Ozbay, K., Yang, H., Bartin, B., Mudigonda, S., 2008. Derivation and Validation of New Simulation-Based Surrogate Safety Measure. Transportation Research Record 2083, 105-113. Pareto, V. 1896. Cours D’Economie Politique. Rouge, Lausanne, Switzerland.

Park, B., Messer, C.J., Urbanik, T., 1999. Traffic signal optimization program for oversaturated conditions: genetic algorithm approach. Transportation Research Record: Journal of the Transportation Research Board 1683, 133-142.

Park, B." B., Messer, C.J., Urbanik II, T., 2000. Enhanced genetic algorithm for signal-timing optimization of oversaturated intersections. Transportation Research Record: Journal of the Transportation Research Board 1727, 32-41.

Park, J.Y., Noland, R.B., Polak, J.W., 2001. Microscopic model of air pollutant concentrations: Comparison of simulated results with measured and macroscopic estimates. Transportation Research Record: Journal of the Transportation Research Board 1750, 64-73.

Park, B., Chen, Y., Hourdos, J., 2011. Opportunities for preventing rear-end crashes: findings from the analysis of actual freeway crash data. Journal of Transportation Safety \& Security 3, 95-107.

Paz, A., \& Chiu, Y. C., 2011. Adaptive traffic control for large-scale dynamic traffic assignment applications. Transportation Research Record: Journal of the Transportation Research Board, 2263(1), 103-112.

Pirdavani, A., Brijs, T., Bellemans, T., Wets, G., 2010. A simulation-based traffic safety evaluation of signalized intersections. Presented at the Proceedings of the Road Safety on Four Continents Conference, Conference Sponsor, pp. 1229-1239.

Planung Transport Verkehr AG, 2010. VISSIM 5.30 User Manual. Karlsruhe, Germany: PTV.

Qian, R., Lun, Z., Wenchen, Y., Meng, Z., 2013. A Traffic Emission-saving Signal Timing Model for Urban Isolated Intersections. Procedia-Social and Behavioral Sciences 96, 2404-2413.

Rakha, H., Ding, Y., 2003. Impact of stops on vehicle fuel consumption and emissions. Journal of Transportation Engineering 129, 23-32. 
Rakha, H., Ahn, K., Trani, A., 2004. Development of VT-Micro model for estimating hot stabilized light duty vehicle and truck emissions. Transportation Research Part D: Transport and Environment 9, 49-74.

Ran, H., 2007. A framework for the determination of weak Pareto frontier solutions under probabilistic constraints. Dissertation, Georgia Institute of Technology.

Reynoso-Meza, G., Sanchis, J., Blasco, X., Herrero, J.M., 2012. Multiobjective evolutionary algorithms for multivariable PI controller design. Expert Systems with Applications 39, 78957907.

Robertson, D., Lucas, C., Baker, R., 1980. Coordinating traffic signals to reduce fuel consumption. TRL Report LR934.Transport and Road Research Laboratory, Crowthorne, Berkshire United Kingdom.

Robles, D., 2012. Optimal signal control with multiple objectives in traffic mobility and environmental impacts. M. Sc. Diss., Royal Institute of Technology (KTH) Stockholm.

Rouphail, N.M., Frey, H.C., Colyar, J.D., Unal, A., 2001. Vehicle emissions and traffic measures: exploratory analysis of field observations at signalized arterials. Presented at the 80th Annual Meeting of the Transportation Research Board, Washington, DC.

Sabra, Z.A., Gettman, D., Henry R.D., Nallamothu, V., 2010. Balancing Safety and Capacity in an Adaptive Signal Control System - Phase 1. Report No. HRT-10-038, Federal Highway Administration, Washington, DC.

Sahoo, S., Albrecht, A.A., 2010. Ranking of microRNA target prediction scores by Pareto front analysis. Computational biology and chemistry 34, 284-292.

Scora, G., Barth, M., 2006. Comprehensive modal emissions model (CMEM), version 3.01. User guide. Centre for Environmental Research and Technology. University of California, Riverside.

Son, H., Kweon, Y.-J., Park, B., 2011. Development of crash prediction models with individual vehicular data. Transportation research part C: emerging technologies 19, 1353-1363.

Srinivas, N., Deb, K., 1994. Muiltiobjective optimization using nondominated sorting in genetic algorithms. Evolutionary computation 2, 221-248.

Stathopoulos, F.G., Noland, R.B., 2003. Induced travel and emissions from traffic flow improvement projects. Transportation Research Record: Journal of the Transportation Research Board 1842, 57-63.

Stevanovic, A., Martin, P.T., and Stevanovic, J., 2007. VISGAOST: VISSIM-based Genetic Algorithm Optimization of Signal Timings. Transportation Research Record 2035, 59-68. 
Stevanovic, A.Z., Martin, P.T., 2008. An Assessment of the Suitability of Microsimulation as a Tool for the Evaluation of Macroscopically Optimized Traffic Signal Timings. Journal of Transportation Engineering ASCE 134(2), 59-67.

Stevanovic, J., Stevanovic, A., Martin, P.T., Bauer, T., 2008. Stochastic optimization of traffic control and transit priority settings in VISSIM. Transportation Research C 16(3), 332-349.

Stevanovic, A., Stevanovic, J., Zhang, K., Batterman, S., 2009. Optimizing Traffic Control to Reduce Fuel Consumption and Vehicular Emissions: Integrated Approach of VISSIM, CMEM, and VISGAOST. Transportation Research Record 1707, 105-113.

Stevanovic, A., Stevanovic, J., Kergaye, C., 2013 (a). Optimization of traffic signal timings based on surrogate measures of safety. Transportation Research Part C: Emerging Technologies $32,159-178$.

Stevanovic, A., Stevanovic, J., and Kergaye, C. (2013) (b). "Green Light Optimized Speed Advisory Systems - Impact of Signal Phasing Information Accuracy." Transportation Research Record, Journal of the Transportation Research Board, No. 2390, 53-59.

Stevanovic, A., Stevanovic, J., Kergaye, C. 2014. Comparative Evaluation of Benefits from Traffic Signal Retiming and Green Light Optimized Speed Advisory Systems. Presented at the $93^{\text {rd }}$ Annual Meeting of the Transportation Research Board, Washington, DC.

Sun, D., Benekohal, R.F., Waller, S.T., 2003. Multi-objective Traffic Signal Optimization Using Non-dominated Sorting Genetic Algorithm. IEEE Intelligent Vehicles Symposium, Piscataway, N.J., 198-203.

Tan, D.M., Alhajyaseen, W.K., Asano, M., Nakamura, H., 2012. Development of Microscopic Traffic Simulation Model for Safety Assessment at Signalized Intersections. Transportation Research Record: Journal of the Transportation Research Board 2316, 122-131.

Yukish, M.A., 2004. Algorithms to identify Pareto points in multi-dimensional data sets. Doctoral dissertation, The Pennsylvania State University.

Yun, I., Park, B., 2005. Stochastic optimization method for coordinated actuated signal systems. Center for Transportation Studies, University of Virginia.

Zhang, L., Yin, Y., Chen, S., 2013. Robust signal timing optimization with environmental concerns. Transportation Research Part C: Emerging Technologies 29, 55-71.

Zhang, Y., Chen, X., Zhang, X., Song, G., Hao, Y., Yu, L., 2009. Assessing effect of traffic signal control strategies on vehicle emissions. Journal of Transportation Systems Engineering and Information Technology 9, 150-155. 
Zhou, S., Yan, X., Wu, C., 2008. Optimization model for traffic signal control with environmental objectives. Presented at the Natural Computation, 2008. ICNC'08. Fourth International Conference on, IEEE, pp. 530-534.

Zitzler, E., Thiele, L., 1999. Multiobjective evolutionary algorithms: a comparative case study and the strength Pareto approach. Evolutionary Computation, IEEE Transactions on 3, 257-271.

Zitzler, E., Thiele, L., Laumanns, M., Fonseca, C.M., Da Fonseca, V.G., 2003. Performance assessment of multiobjective optimizers: An analysis and review. Evolutionary Computation, IEEE Transactions on 7, 117-132.

Zou, J., Mueller, D., Graeb, H., Schlichtmann, U., 2007. Pareto-front computation and automatic sizing of cpplls. Presented at the Quality Electronic Design, 2007. ISQED'07. 8th International Symposium on, IEEE, pp. 481-486. 
Multi-Criteria Optimization of Traffic Signals: Mobility, Safety, and Environment

Aleksandar Stevanovic, Jelka Stevanovic, Jaehyun So, and Marija Ostojic

Manuscript Number: TRC-D-14-00543

FIGURE 1 VISSIM-CMEM-VISGAOST Integration.

FIGURE 2 Case-study Network.

FIGURE 3 Calibration and Validation of Simulation Model.

FIGURE 4 Signal Timings in 3-D and 2-D Space: Broad Search.

FIGURE 5 Closer Look of Pareto Fronts under Broad Search.

FIGURE 6 Signal Timings in 3-D and 2-D Space: Constrained Search.

FIGURE 7 Closer Look of Pareto Fronts under Constrained Search.

FIGURE 8 Representative Signal Timings for Externalities and Throughputs. 


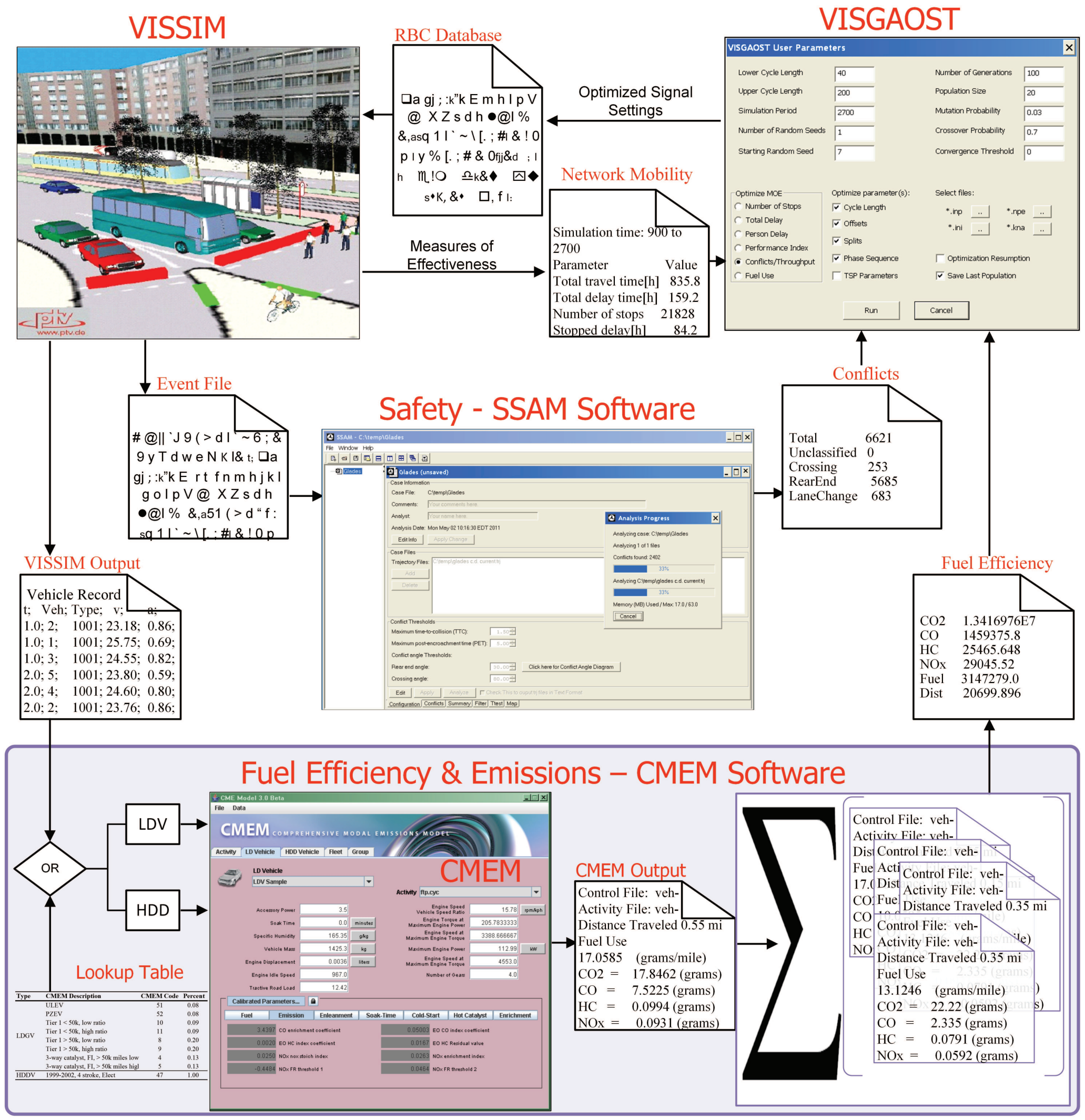


Figure 3

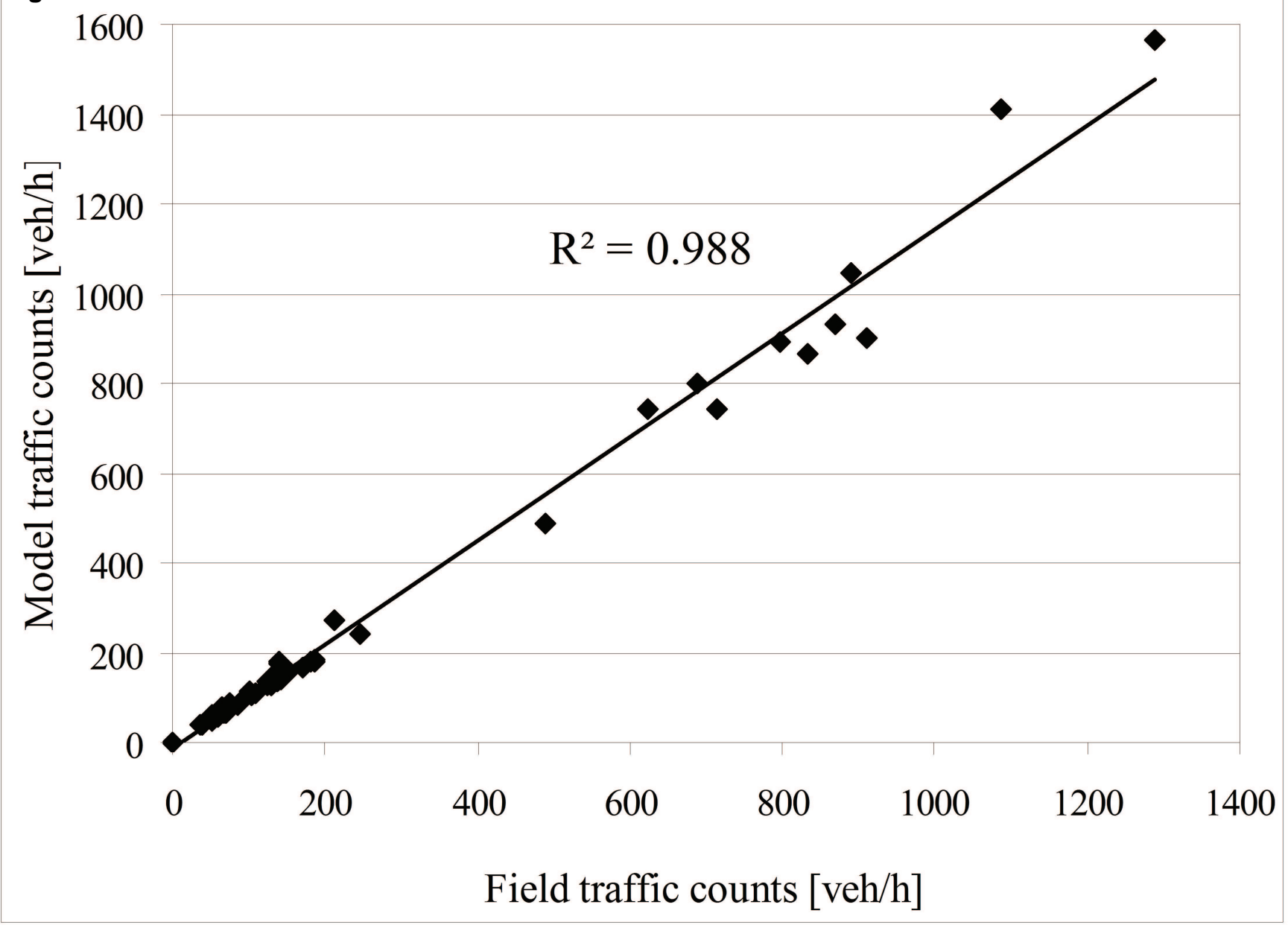

A) Calibration Results - Turning Movement Counts

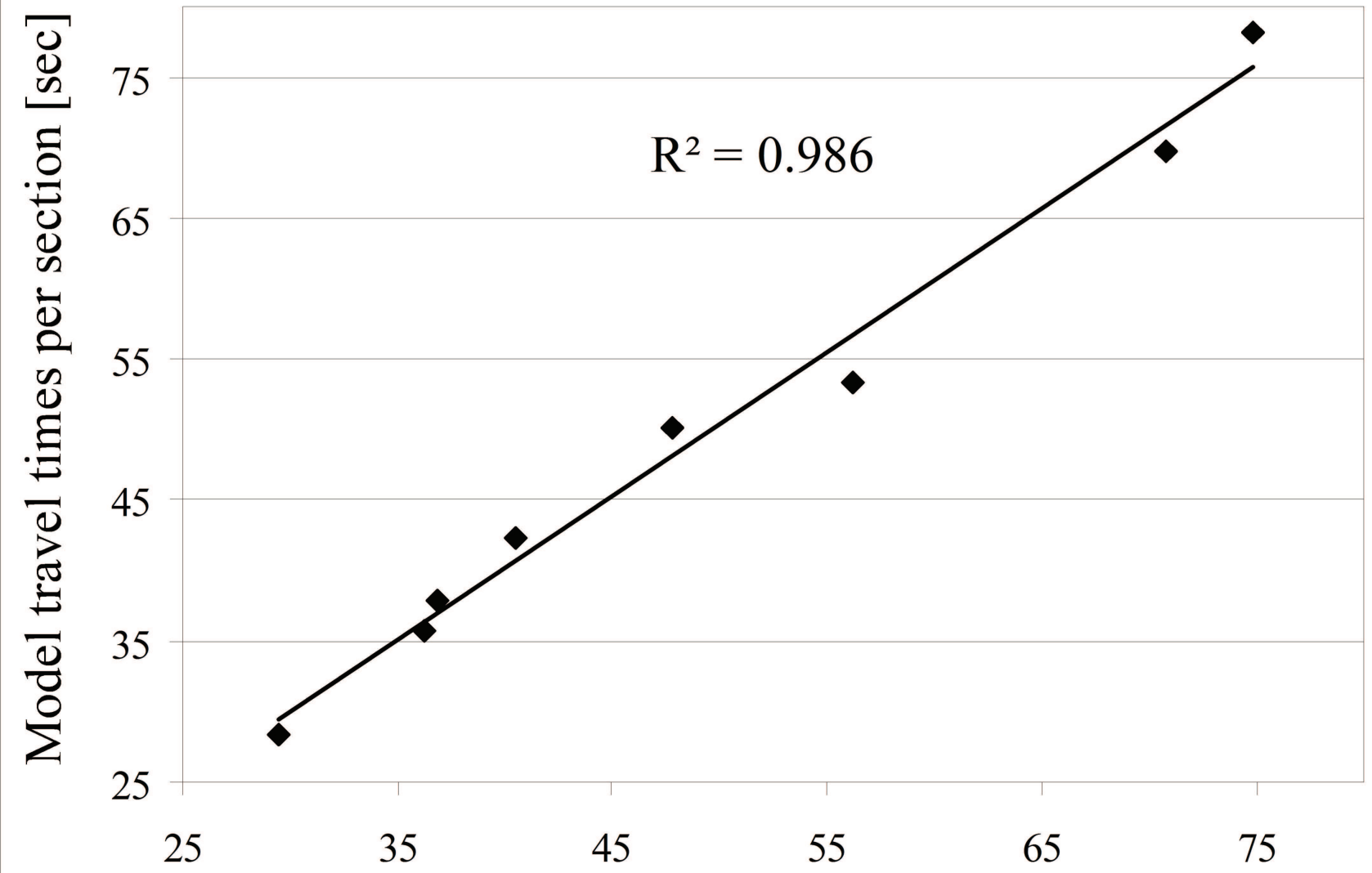

Field travel times per section [sec]

B) Validation Results - Travel Times 


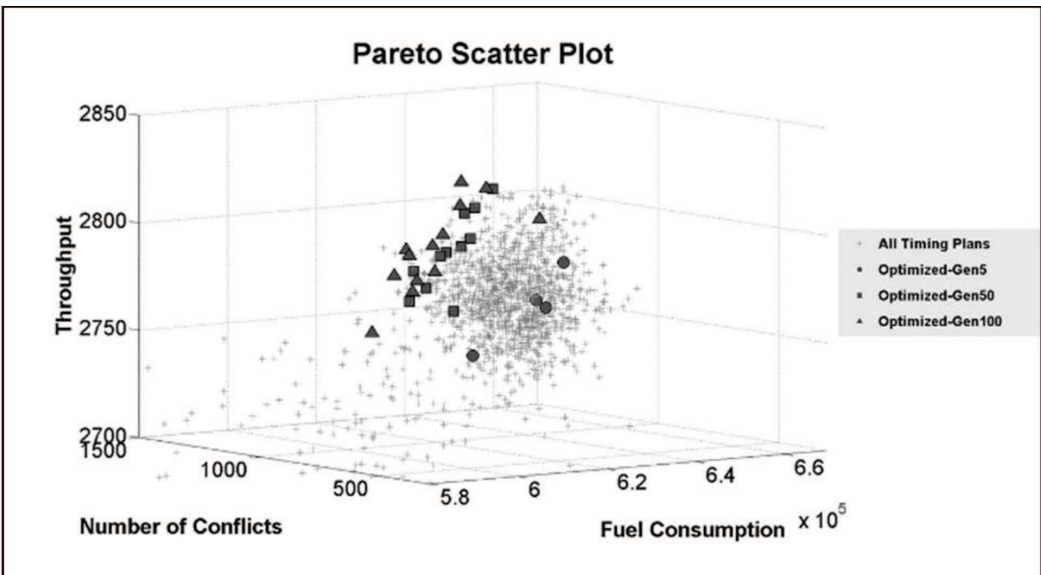

A) Signal Timings in 3-D Space: A Closer Look

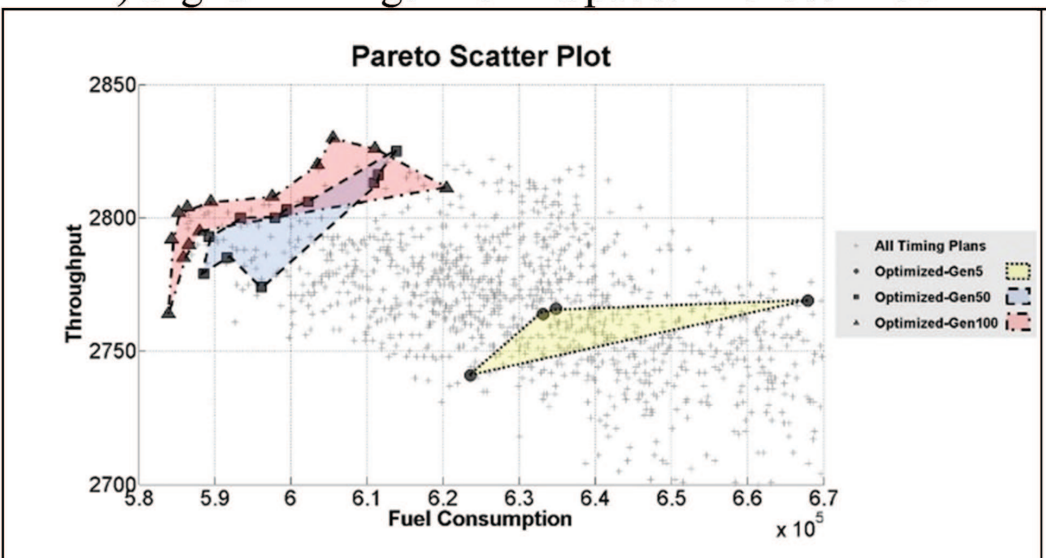

C) 3-D Pareto Fronts in Mobility-Environ. 2-D Space

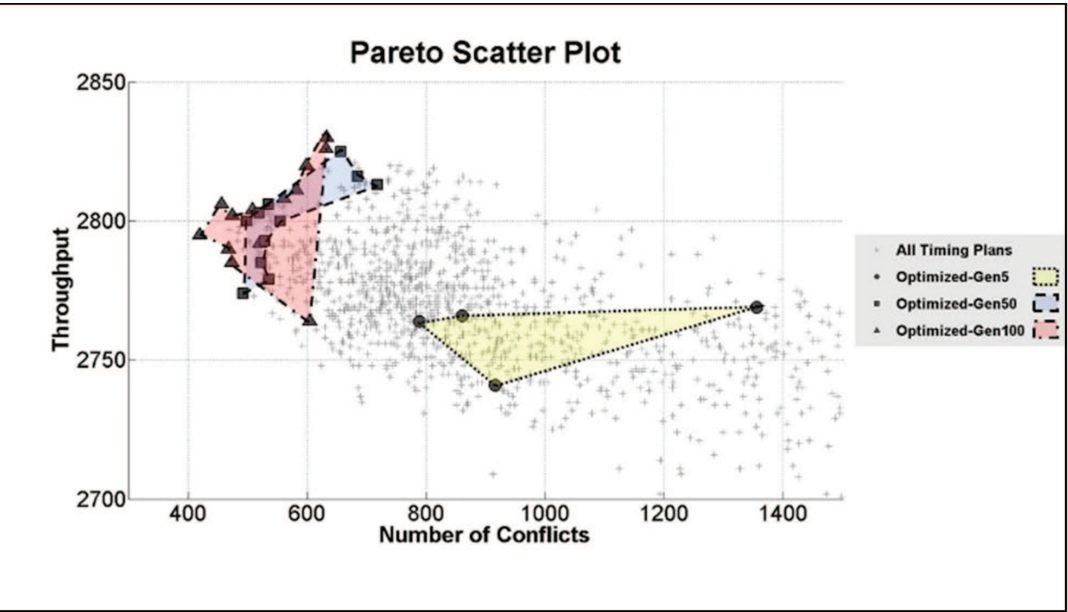

B) 3-D Pareto Fronts in Mobility-Safety 2-D Space

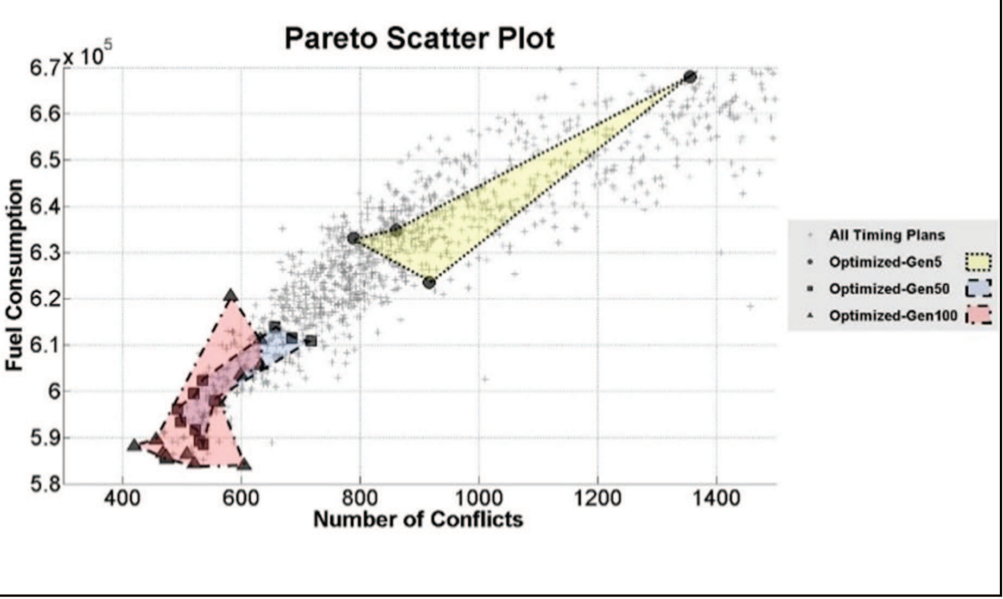

D) 3-D Pareto Fronts in Environment-Safety 2-D Space 


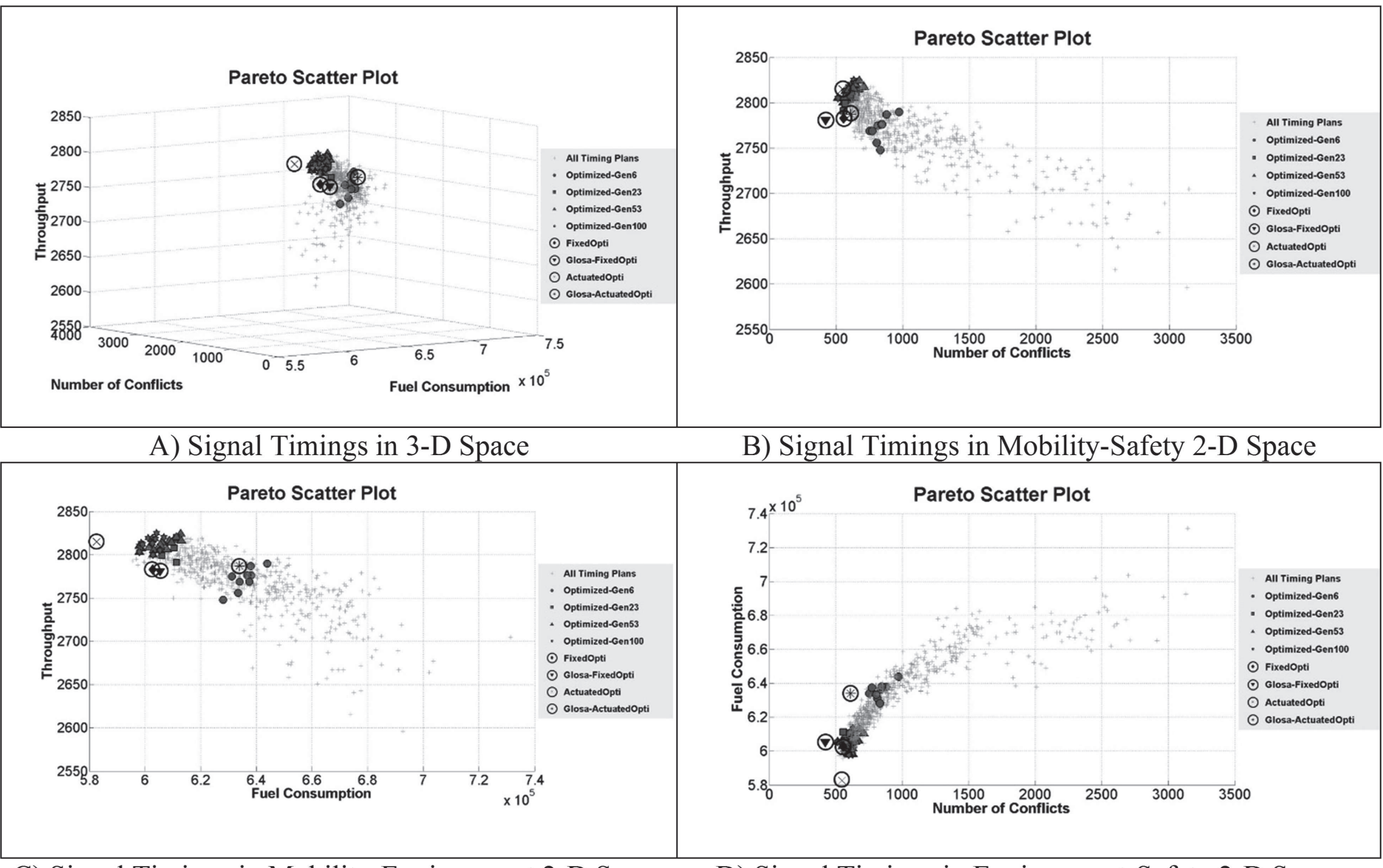




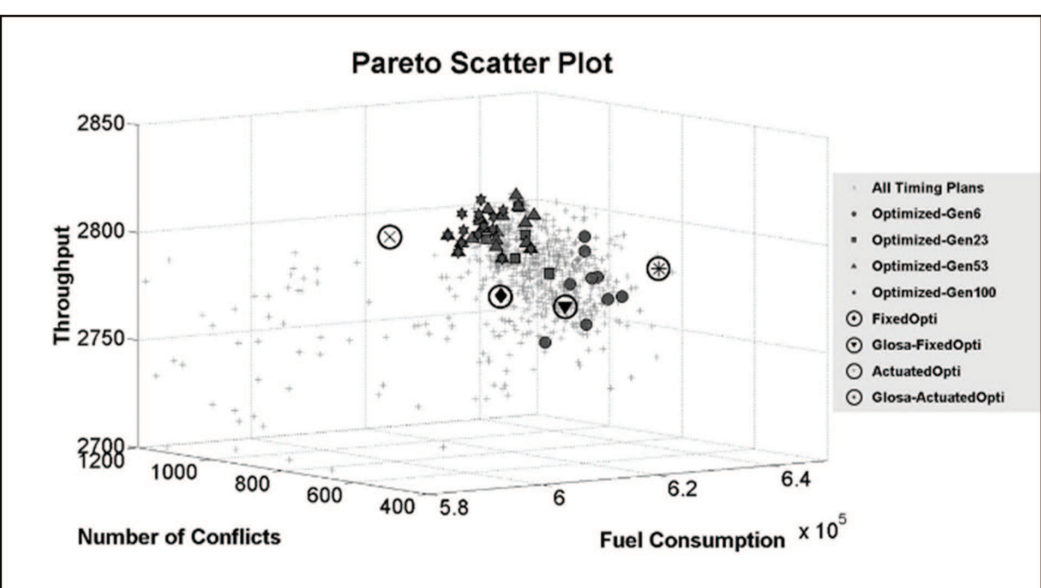

A) Signal Timings in 3-D Space: A Closer Look

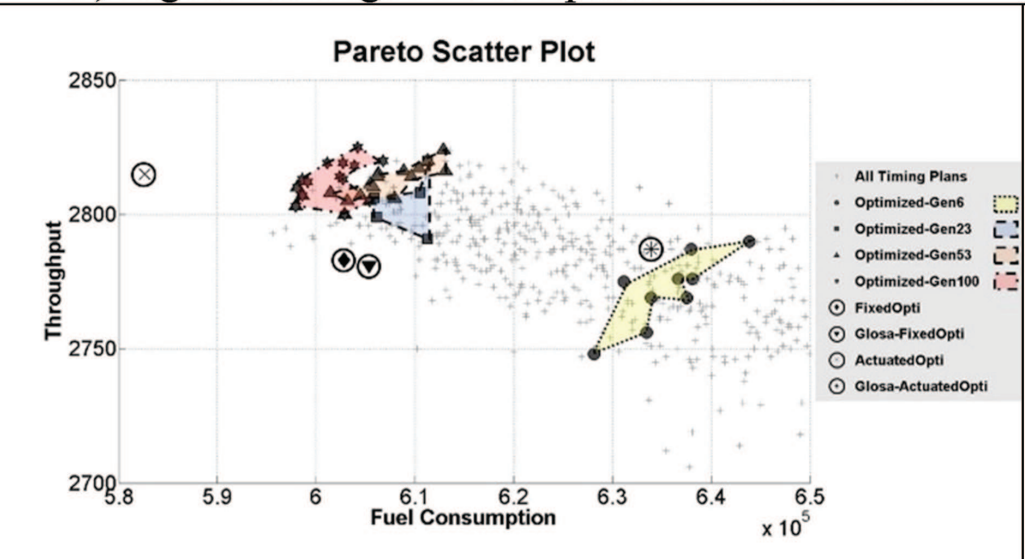

C) 3-D Pareto Fronts in Mobility-Environ. 2-D Space

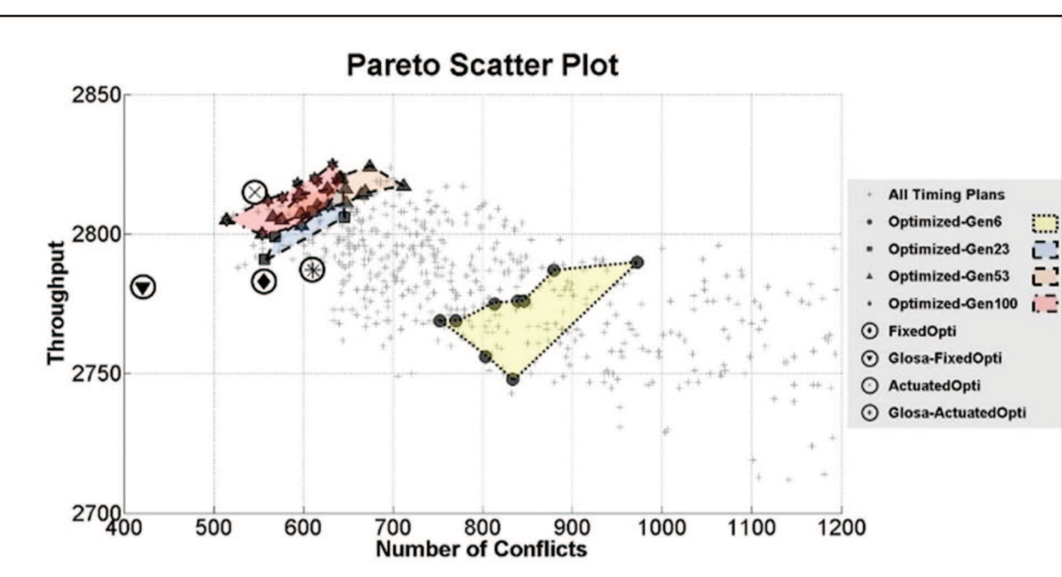

B) 3-D Pareto Fronts in Mobility-Safety 2-D Space

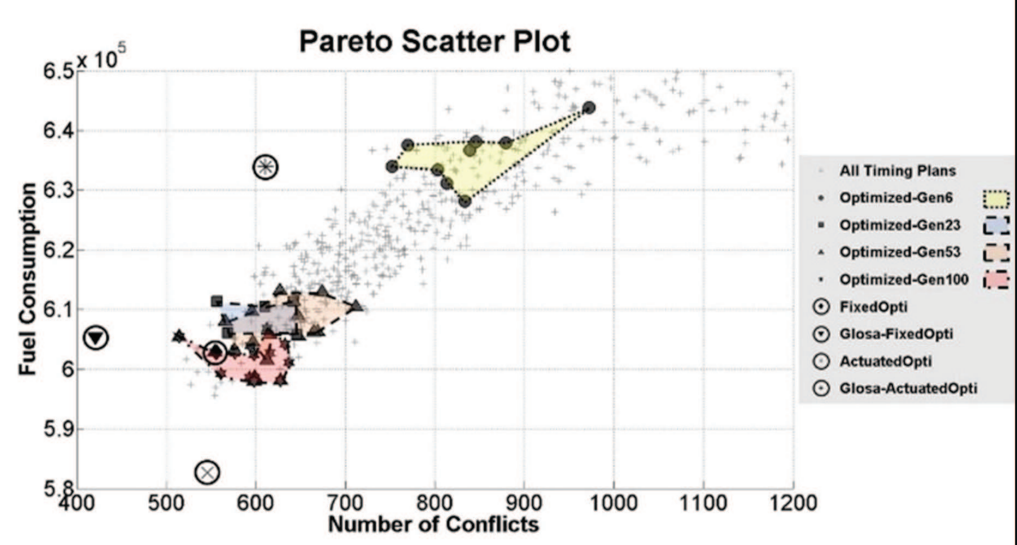




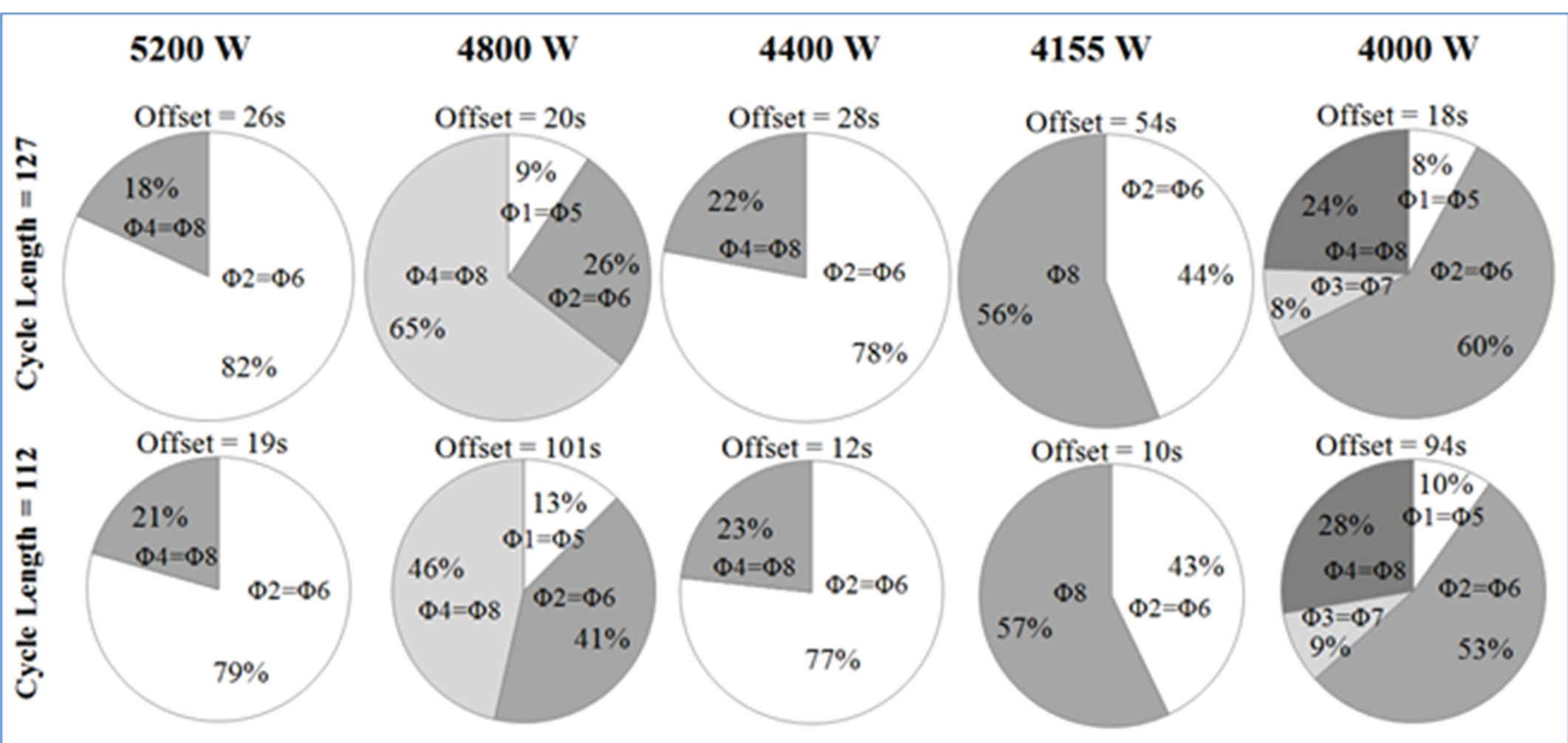


Table 1 Performance of Signal Timings from the Two 3-D Pareto Optimizations.

\begin{tabular}{cccc}
\hline $\begin{array}{c}\text { Signal } \\
\text { Timing Plan }\end{array}$ & $\begin{array}{c}\text { Throughput } \\
\text { Trips/30 min }\end{array}$ & $\begin{array}{c}\text { CMEM Fuel } \\
\text { [grams] }\end{array}$ & $\begin{array}{c}\text { SSAM } \\
\text { \# of Conflicts }\end{array}$ \\
\hline 1 & 2764 & 583898 & 605 \\
2 & 2785 & 585872 & 474 \\
3 & 2790 & 586588 & 468 \\
4 & 2792 & 584318 & 521 \\
5 & 2795 & 588011 & 420 \\
6 & 2802 & 585273 & 475 \\
7 & 2804 & 586392 & 509 \\
8 & 2806 & 589516 & 457 \\
9 & 2808 & 597559 & 562 \\
10 & 2810 & 598095 & 628 \\
11 & 2812 & 599173 & 561 \\
12 & 2813 & 602727 & 577 \\
13 & 2813 & 598632 & 593 \\
14 & 2814 & 602414 & 598 \\
15 & 2818 & 603875 & 594 \\
16 & 2819 & 601128 & 637 \\
17 & 2819 & 602712 & 616 \\
18 & 2820 & 603515 & 599 \\
19 & 2825 & 604217 & 633 \\
20 & 2826 & 611086 & 632 \\
21 & 2830 & 605592 & 633 \\
\hline Highest & & & $<3 \%$ \\
Difference & $<3 \%$ & $<4.5 \%$ & \\
\hline
\end{tabular}

Shaded signal timing plans are those from not-constrained optimization 